\title{
Is Lupus Anticoagulant a Significant Feature of COVID-19? A Critical Appraisal of the Literature
}

\author{
Emmanuel J. Favaloro, PhD, FFSc (RCPA) ${ }^{1,2}$ Brandon Michael Henry, MD ${ }^{30}$ Giuseppe Lippi, MD ${ }^{4(0)}$
}

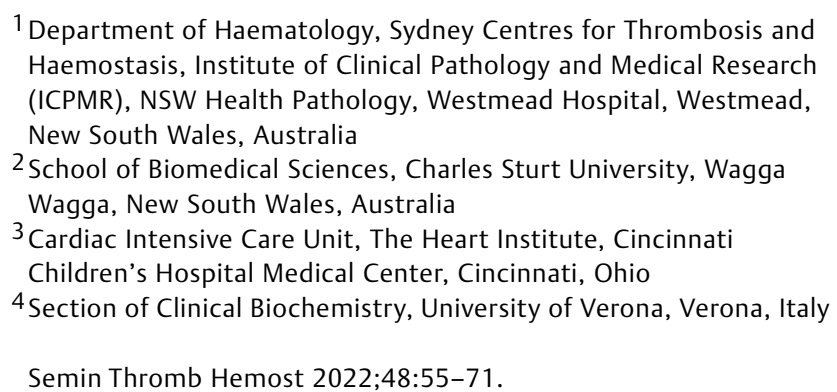

Address for correspondence Emmanuel J. Favaloro, PhD, FFSc (RCPA), Department of Haematology, Institute of Clinical Pathology and Medical Research (ICPMR), Westmead Hospital, Westmead, New South Wales, 2145 Australia

(e-mail: emmanuel.favaloro@health.nsw.gov.au).

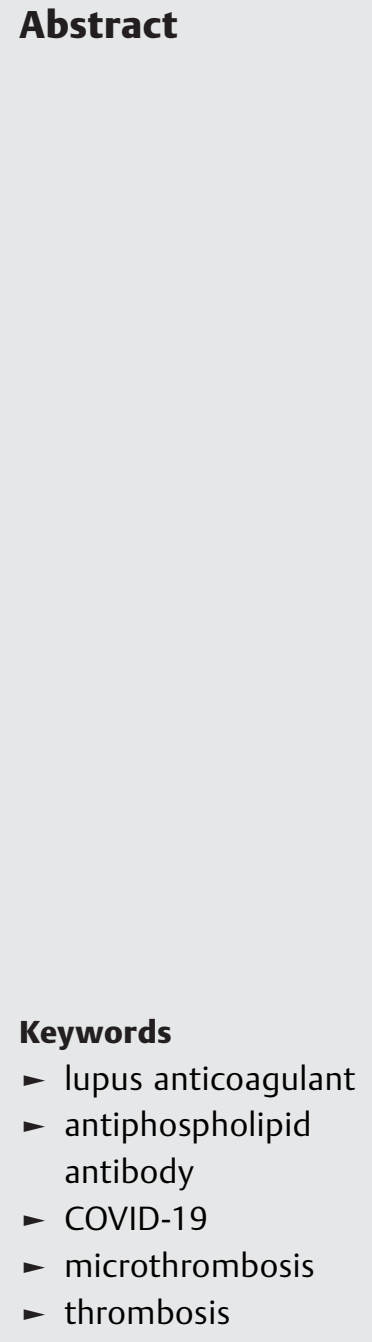

The term "lupus anticoagulant (LA)" identifies a form of antiphospholipid antibodies (aPLs) causing prolongation of clotting tests in a phospholipid concentration-dependent manner. LA is one of the laboratory criteria identified in patients with antiphospholipid (antibody) syndrome (APS). The presence of LA in patients with APS represents a significant risk factor for both thrombosis and pregnancy morbidity. There have been several reports of similarities between some of the pathophysiological features of COVID-19 and APS, in particular the most severe form, catastrophic APS. There have also been many reports identifying various aPLs, including LA, in COVID-19 patients. Accordingly, a very pertinent question arises: "Is LA a feature of COVID-19 pathology?" In this review, we critically appraise the literature to help answer this question. We conclude that LA positivity is a feature of COVID-19, at least in some patients, and potentially those who are the sickest or have the most severe infection. However, many publications have failed to appropriately consider the many confounders to LA identification, being assessed using clot-based assays such as the dilute Russell viper venom time, the activated partial thromboplastin time (aPTT), and the silica clotting time. First, most patients hospitalized with COVID-19 are placed on anticoagulant therapy, and those with prior histories of thrombosis would possibly present to hospital already on anticoagulant therapy. All anticoagulants, including vitamin $\mathrm{K}$ antagonists, heparin (both unfractionated heparin and low-molecular-weight heparin), and direct oral anticoagulants affect these clot-based assays. Second, C-reactive protein (CRP) is highly elevated in COVID-19 patients, and also associated with severity. CRP can also lead to false-positive LA, particularly with the aPTT assay. Third, persistence of aPL positivity (including LA) is required to identify APS. Fourth, those at greatest risk of thrombosis due to $\mathrm{APL}$ are those with highest titers or multiple positivity. Most publications either did not identify anticoagulation and/or CRP in their COVID-19 cohorts or did not seem to account for these as possible confounders for LA detection. Most publications did not assess for aPL persistence, and where persistence was checked, LA appeared to represent transient aPL. Finally, high titer aPL or multiple aPL published online June 15, 2021
Issue Theme Maintaining Hemostasis and Preventing Thrombosis in COVID-19-Part III; Guest Editors: Emmanuel J. Favaloro, PhD, FFSc (RCPA) and Giuseppi Lippi, MD (c) 2021. Thieme. All rights reserved. Thieme Medical Publishers, Inc., 333 Seventh Avenue, 18th Floor, New York, NY 10001, USA
DOI https://doi.org/ 10.1055/s-0041-1729856. ISSN 0094-6176. 
positivity were in the minority of COVID-19 presentations. Thus, at least some of the reported LAs associated with COVID-19 are likely to be false positives, and the relationship between the detected $\mathrm{APL} / \mathrm{LA}$ and COVID-19-associated coagulopathy remains to be resolved using larger and better studies.

The term "lupus anticoagulant (LA)" identifies a form of antiphospholipid antibodies (aPLs) causing prolongation of clotting tests in a phospholipid concentration-dependent manner. LA is one of the laboratory criteria identified in patients with antiphospholipid (antibody) syndrome (APS). ${ }^{1,2}$ The term "lupus anticoagulant" is actually a double misnomer, as it represents neither a specific feature of systemic lupus erythematosus (SLE) nor an "anticoagulant."3,4 Indeed, the presence of LA in patients with APS represents a significant risk factor for both thrombosis and pregnancy morbidity. ${ }^{1,2,5}$ Thus, patients with LA positivity are considered to carry a theoretical risk of a thrombophilia-like disorder.

COVID-19 (coronavirus disease 2019) has been declared a pandemic, and is caused by infection with SARS-CoV-2 (severe acute respiratory syndrome coronavirus 2). Thought to have originated in Wuhan, China, in late 2019, COVID-19 is now well-known to reflect a prothrombotic disorder, ${ }^{6}$ and thrombosis in various forms affects a high proportion of severely infected individuals. For example, a recent metaanalysis has suggested a venous thrombosis rate, including deep vein thrombosis (DVT) and pulmonary embolism (PE) of close to $30 \%$ in patients with severe COVID-19. ${ }^{7}$ Acute myocardial ischemia (infarction) and cerebrovascular accidents may also develop in as many as 8 and 3\% of COVID-19 patients needing intensive care, ${ }^{8}$ while systemic coagulopathy and disseminated intravascular coagulation may onset in as many as $7 \%$ of such patients. ${ }^{9}$ There is also evidence of microthrombosis in multiple organs including lungs, kidneys, and liver, only identifiable on autopsy, in patients who have died due to COVID-19. ${ }^{10-13}$ Anticoagulant therapy is therefore routinely applied to nearly all patients hospitalized with COVID-19.

There have been several reports of similarities between some of the pathophysiological features of COVID-19 and APS, in particular the most severe form, catastrophic APS (CAPS). ${ }^{14-16}$ Indeed patients with COVID-19 appear to fulfill the main clinical diagnostic criteria for CAPS: evidence of involvement in three or more organs, development of manifestations simultaneously or in less than a week, and confirmation by histopathology of small vessel occlusion in at least one organ. ${ }^{16}$ There have also been many reports identifying various aPL, including LA, in COVID-19 patients. The search for aPL in COVID-19 may have been sparked by an early publication by Zhang et al $2020^{17}$ in the New England Journal of Medicine.

Given the above, some relevant questions would naturally arise. Given (1) LA is associated with thrombosis, (2) patients with COVID-19 suffer thrombosis, (3) some aspects of COVID-19 pathology strongly resemble CAPS, and (4) aPLs, including LA, have been identified in COVID-19 in several studies, perhaps the most pertinent question: "Is LA a feature of COVID-19 pathology?" In this review, we critically appraise the literature to help answer this question.

\section{Thrombosis-Associated LA versus Laboratory-Detected LA}

Before we specifically address this question, some additional pertinent background information is required. First, despite an association of LA and other aPL with thrombosis risk in APS and in other potential autoimmune diseases, the presence of a laboratory-detected LA or/and other aPLs per se do not, in themselves, reflect a prothrombotic risk factor, even if persistent, and do not warrant pharmacological intervention, ${ }^{18,19}$ except perhaps for those with high titer aPL and multiple positivity. ${ }^{20,21}$ Indeed, laboratory-detected LA is often found in asymptomatic patients, many of who will never develop thrombosis. For example, laboratory-detected LA often arises as a result of a follow-up to an unexpected prolonged activated partial thromboplastin time (aPTT). This may occur, for example, when an aPTT is ordered as a screening assay for preoperative bleeding risk, ${ }^{22}$ and should an LA-sensitive APTT reagent be used for the test. This "chance" finding may cause some angst in the requesting clinical team, who may then be tempted to cancel or postpone surgery, and notwithstanding expert recommendations to not use the aPTT for such purpose, ${ }^{22}$ or else to preferentially use an LA-insensitive aPTT reagent for general screening purposes, and reserving LA sensitive aPTT regents for formal LA investigations in (for example) APS workups. ${ }^{23}$

There are many other reasons why a laboratory-identified LA may not reflect a prothrombotic marker, in particular due to preanalytical or analytical issues causing false-positive LA test results. The presence of anticoagulants, in particular, can give rise to false LA findings. This may even reflect a circular argument of sorts, as patients with thrombosis, or at risk of thrombosis, including those with APS, may be placed on anticoagulant therapy for thrombosis treatment or prevention. If the LA tests are performed while the patient is undergoing anticoagulant therapy, then there is a great risk of a false-positive LA. The possibility of a false-positive LA is true for most anticoagulants, in part depending on how the LA tests are performed. This is expanded on later.

\section{Lupus Anticoagulant Testing Guidelines}

There are three groups who have recently provided guidelines on LA testing, the International Society on Thrombosis 
and Haemostasis (ISTH), the Clinical and Laboratory Standards Institute (CLSI), and the British Committee for Standards in Haematology (BCSH). The ISTH has prepared a series of such guidelines, starting in $1991^{24}$ and last updated in $2020,{ }^{23}$ although most laboratories are probably still using and referring to the 2009 guidelines. ${ }^{25}$ The BCSH published their guidance in 2012, ${ }^{26}$ and the CLSI published their guidance in 2014. ${ }^{27}$ All this historical context has some relevance to LA testing in 2021, in particular as related to anticoagulant effects. The 2009 ISTH and 2012 BCSH guidelines were published when the main anticoagulants were vitamin K antagonists (VKAs; such as warfarin) and heparins, including unfractionated heparin (UFH) and low-molecularweight heparin (LMWH). The presence of these anticoagulants in the blood of patients on therapy taken for tests can affect clotting assays, including those for LA. Thus, these guidelines attempted to address strategies for assessment of LA in the presence of these anticoagulants, but did not cover the direct oral anticoagulants (DOACs), as these had not yet been introduced into clinical practice.

\section{Assays Used for LA Detection/Exclusion and Anticoagulant Interference}

The main assays used for LA identification/exclusion are the dilute Russell viper venom time (dRVVT) and the aPTT. ${ }^{28,29}$ The silica clotting time (SCT) represents a form of aPTT assay marketed by at least one of the major commercial providers, and is becoming increasingly popular for assessing LA, sometimes instead of the "classical" aPTT. ${ }^{28}$ The strategies employed for countering anticoagulant effects in LA investigations, as considered in the earlier LA test guidelines, ${ }^{25,26}$ include (1) the addition of a heparin neutralizer in dRVVT reagents, capable of neutralizing therapeutic heparin levels up to approximately $1 \mathrm{U} / \mathrm{mL}$ and (2) the use of mixing studies to eliminate or dampen the effects of VKAs, which essentially create "factor deficiencies" of factors II, VII, IX, and X. Thus, therapeutic heparin levels should not affect the dRVVT, but will affect the aPTT, which in essence is used in many laboratories to monitor UFH therapy. Heparin will also affect the SCT (unless the reagent contains a heparin neutralizer). The commercial SCT reagents in most common use do not contain any such heparin neutralizers. There may also be a common misconception that LMWH does not affect the LA tests (dRVVT, aPTT, or the SCT). Like UFH, LMWH should not affect the dRVVT unless the level is supratherapeutic, and exceeds the heparin neutralizing capacity of the reagents in use. Similarly, as LMWH comprises mostly anti-Xa activity, in contrast to UFH which expresses mostly anti-Ila activity, LMWH will have a reduced effect on aPTT and SCT compared with UFH. However, LMWH will prolong both SCT and aPTT in a concentration-dependent manner, especially when therapeutic levels are exceeded. Finally, VKAs will affect dRVVT, aPTT, and SCT, given effects on FII, FVII, FIX, and FXI. Although mixing of patient plasma with normal plasma was identified as an early way of "normalizing" the VKA effect, and making both dRVVT and aPTT test results, when performed as directed by the guidelines, more specific for
$\mathrm{LA}^{25}$ this is no longer recommended in the most recent ISTH guidelines, ${ }^{23}$ since, in theory, false-positive and false-negative LA findings may ensue.

The situation with anticoagulant interference in LA testing magnified considerably with the advent of the new/novel oral anticoagulants or DOACs. These anticoagulant agents affect all the LA clot-based assays (e.g., aPTT, dRVVT, and $\mathrm{SCT}$ ), ${ }^{30-33}$ and since they are "inhibitors" (to either factor IIa or Xa), mixing samples containing DOACs with normal plasma only partially abrogates their effects. Moreover, unlike the case for heparins, DOAC neutralizers ${ }^{34}$ have yet to be formally introduced into commercial dRVVT reagents. Although some of these compounds are now otherwise commercially available, they are not often employed in laboratories, nor has their effect been fully assessed in this context. As noted, the $2009 \mathrm{ISTH}^{25}$ and $2012 \mathrm{BCSH}^{26}$ guidelines were published before the advent of the DOACs, and thus did not provide any guidance for LA testing in their presence. The CLSI guideline ${ }^{27}$ was published as the DOACs were emerging, and thus noted that these had an effect on LA tests; here, the "simple" recommendation was to avoid testing of LA in patients being treated by DOACs.

This is, of course, wishful thinking, and clinicians often ignore such guidance. The situation may go like this-a patient has a thrombosis and is quickly placed on an anticoagulant, and subsequently there is a desire to investigate the cause of the thrombosis. Does the patient have a thrombophilia, for example? Will they need to be on extended anticoagulation therapy? Do they have LA? And thus, tests are often requested on patients who have already started on anticoagulant therapy, despite recognition that the presence or absence of one or more thrombophilic conditions will generally have no impact on therapeutic management in the short term (i.e., within 2-3 months).

\section{COVID-19-A Prothrombotic Condition}

Fast forward to 2020, and the world is in the grips of the COVID-19 pandemic. At the time of this writing, COVID-19 has infected over 120 million people worldwide and has reportedly been responsible for over 2.5 million deaths. ${ }^{35}$ COVID-19 is now well-known to reflect a prothrombotic disorder, ${ }^{6}$ with various forms of thrombosis implicated in the pathogenesis and morbidity/mortality of infected individuals. A high proportion of individuals (close to $30 \%$ in patients with severe COVID-19) suffer from venous thromboembolism, including DVT and PE. ${ }^{7}$ Acute myocardial ischemia (infarction), cerebrovascular events, and arterial thrombosis may also develop in a smaller proportion of COVID-19 patients, especially those needing intensive care. ${ }^{8,9}$ There is also evidence of microthrombosis in multiple organs including lungs, kidneys, and liver. ${ }^{10-13}$

As part of a search to investigate the mechanisms that promote thrombosis in COVID-19, many tests of hemostasis have been investigated in patients suffering from this disease. Indeed, many tests of hemostasis are abnormal in patients with COVID-19. ${ }^{36,37}$ Moreover, COVID-19 appears to affect all aspects of hemostasis, 
including primary hemostasis (endothelium, platelets, von Willebrand factor), secondary hemostasis/coagulation, and fibrinolysis. $^{38-43}$

\section{Literature Search}

To give some additional background to this narrative review, we searched the PubMed database (https://pubmed.ncbi.nlm. nih.gov) using various iterations of COVID-19 together with various iterations of LA and (anti)phospholipid antibodies. An initial search performed on February 22, 2021, was later updated to be current as of March 6, 2021. Of over 200 separate articles identified by this search, we then excluded general reviews, commentaries, and articles otherwise found to be irrelevant to the topic. We also excluded single case reports, but small case series were included.

\section{Results of the Literature Review-Is LA Present in COVID-19?}

A summary of the literature arising from our search is given in -Table 1. There was a large body of publications. ${ }^{17,44-69}$ Although additional relevant articles are likely available in the literature, the captured articles are sufficient for us to critically review the literature. We are focusing here on LA. Although several articles reported on aPL other than, or in addition to, LA, these will largely not be assessed in the current review, and instead are the proposed topic of a second forthcoming review. There was a wide variety of methods employed to identify LA ( - Table 1), but often, the methodology was not even reported. There was a wide variety also in COVID-19 case numbers and type, including in some reports "severe" COVID-19, using a variety of definitions (i.e., needing mechanical ventilation or intensive care; mortality).

Of interest, LA was not always detected in patients with COVID-19, as some studies clearly reported "no LA" or very few cases of LA in their patient cohort (-Table 1). However, many publications instead reported LA in a large proportion of their COVID-19 cohorts, in some cases more than $80 \%$. This seems to identify a dichotomy of opinions around the presence of LA in COVID-19. To put a graphical perspective to the data, - Fig. 1 plots the findings from the literature identified in - Table 1 according to percentage positive for LA versus number of investigated cases. There is no obvious pattern.

One of the earliest reports on the presence of aPL in COVID-19 was by Zhang et al ${ }^{17}$ who published their findings in the New England Journal of Medicine. This was a case series report of three patients with COVID-19 in ICU who suffered serious sequelae including multiple infarcts. Interestingly, although aPLs were detected in all three patients, LA was not found in any of the patients. Nevertheless, this study no doubt prompted a wider search for aPL, including LA, in subsequent COVID-19 cohorts. This study could be criticized in several ways. First, the methodology used for aPL detection was not identified, nor were the levels of identified aPL (whether high or low). Persistence of aPL was also not

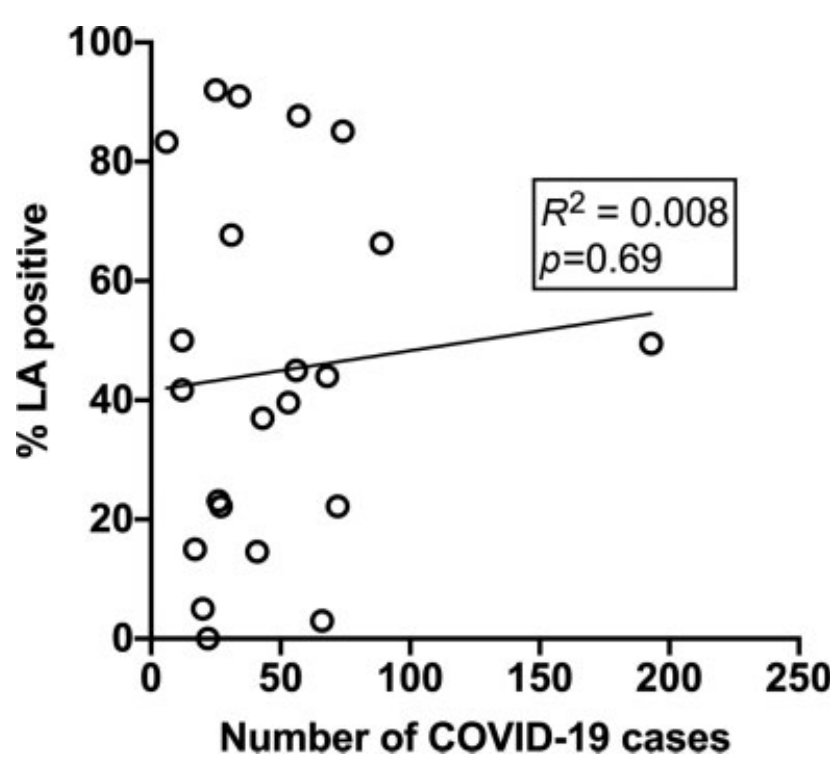

Fig. 1 The relationship between COVID-19 case numbers reported in the literature and the proportional identification of lupus anticoagulant (LA) positive cases.

evaluated. As the study focused on a particular small group of COVID-19 patients, there was also clear patient selection bias. In other words, the study focused on three patients with serious clinical sequelae who also happened to have aPL. There was no evidence of cause or effect. To take a dichotomous perspective, the first article that we identified as reporting on COVID-19 in this arena was from Yasri and Wiwanitkit. ${ }^{44}$ These workers used data collected "according to public official report of $\mathrm{CDC}$ of Thailand, the second country in the timeline of this novel coronavirus outbreak" and identified that APS was rare in COVID-19. From the accumulated 2,369 COVID-19 patients (April 8, 2020) with 30 deaths, only 1 patient $(0.04 \%$ ) had been identified with APS.

It can also be noted that some researchers investigating aPL activity in COVID-19 purposely did not look for LA because they recognized the confounders. For example, although they investigate for aPL, Galeano-Valle et al $^{70}$ purposely did not assess LA "since testing is not recommended in acutely ill patients and under anticoagulant therapy." As another example, Tang $^{49}$ correctly noted that both the ISTH and CLSI urge caution when interpreting LA results in patients receiving anticoagulants. Tang further correctly surmised "Given common use of LMWH and UFH for thromboprophylaxis in COVID-19 inpatients, false-positive results resulting from interference of these anticoagulants may be an important reason for the high positive rate of LA" otherwise found by others, especially when this preanalytical issue is not properly addressed.

\section{Selection Bias in the Literature}

One could hypothesize that the reported incidence of COVID19-associated LA would be higher in small cases series due to potential selection bias, as identified previously for the 


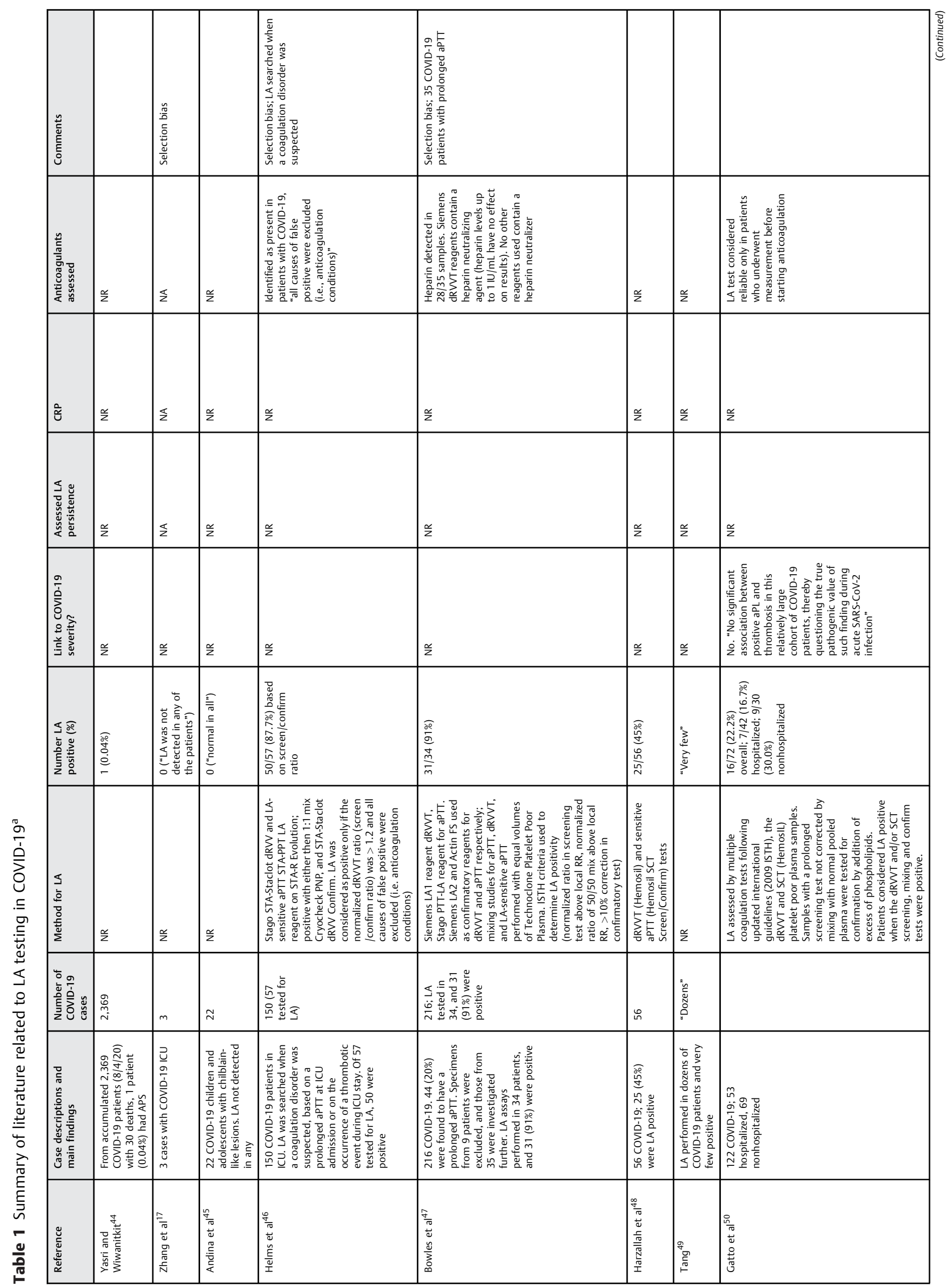


60 Lupus Anticoagulant and COVID-19 Favaloro et al.

\begin{tabular}{|c|c|c|c|c|c|}
\hline & 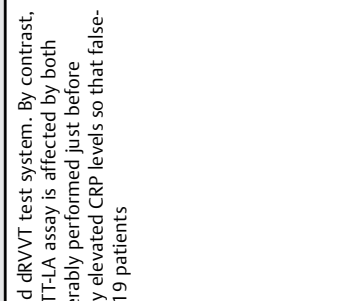 & & 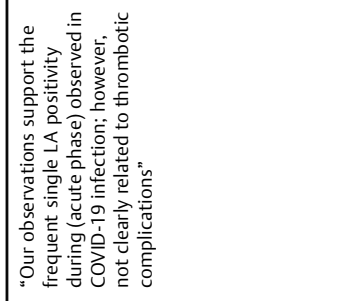 & 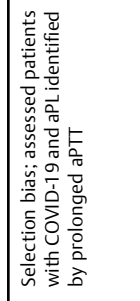 & 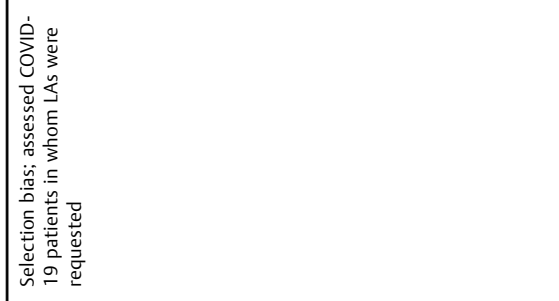 \\
\hline 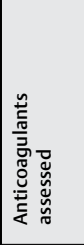 & 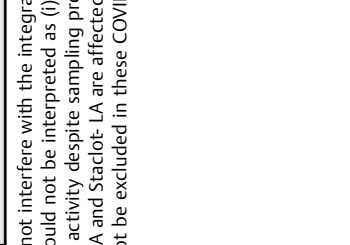 & 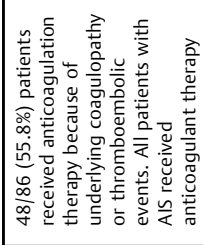 & 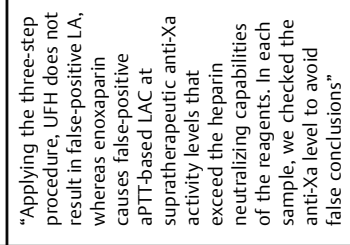 & $\frac{\approx}{z}$ & 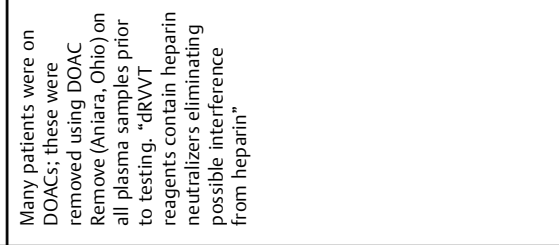 \\
\hline 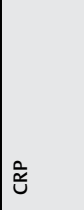 & 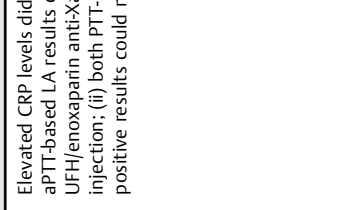 & $\stackrel{\alpha}{z}$ & 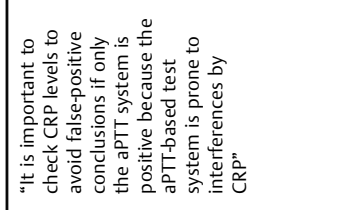 & $\frac{\infty}{2}$ & 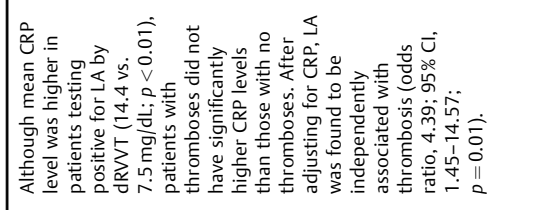 \\
\hline 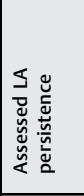 & $\frac{\alpha}{2}$ & $\stackrel{\approx}{z}$ & 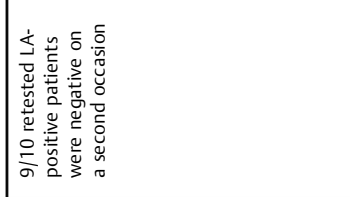 & 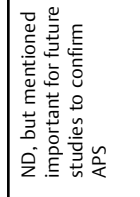 & 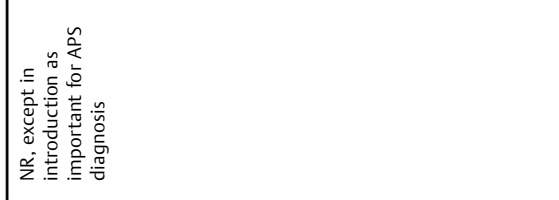 \\
\hline 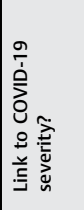 & 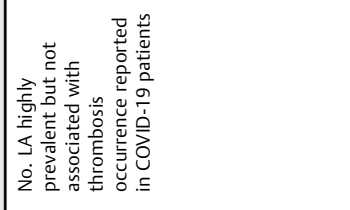 & 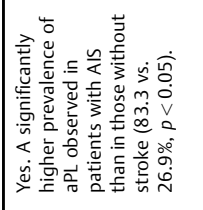 & 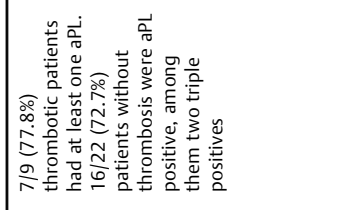 & $\frac{\varkappa}{z}$ & 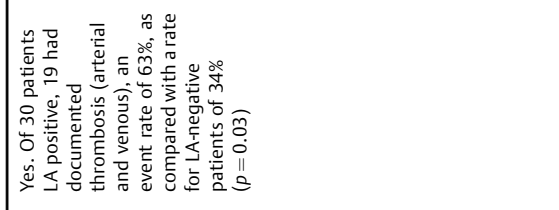 \\
\hline 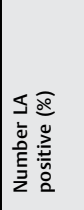 & 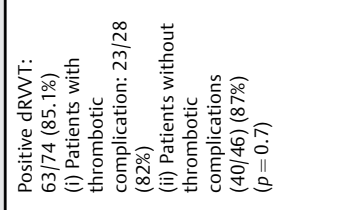 & 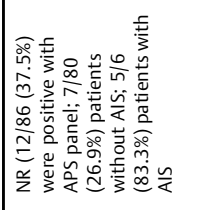 & 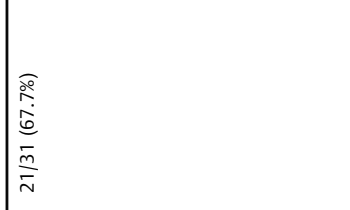 & 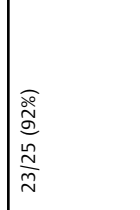 & 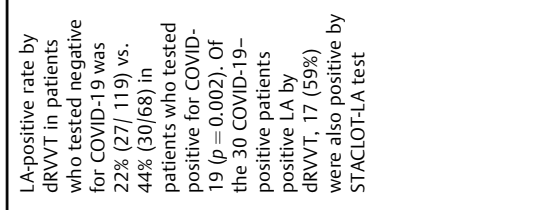 \\
\hline 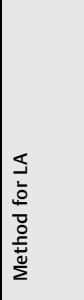 & 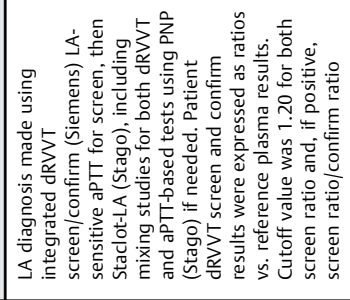 & 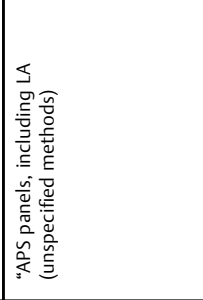 & 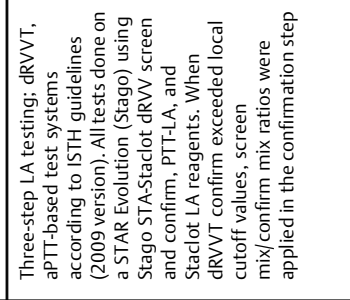 & 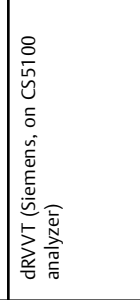 & 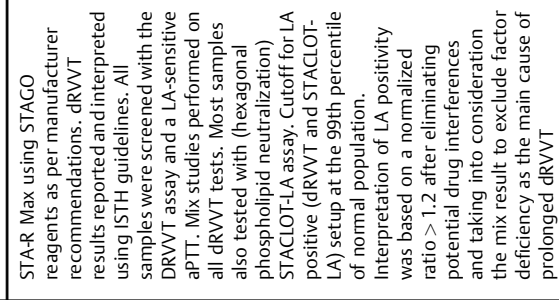 \\
\hline 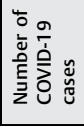 & I & $\infty$ & $\bar{m}$ & $\stackrel{\sim}{2}$ & $\infty$ \\
\hline 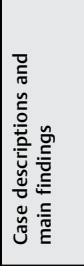 & 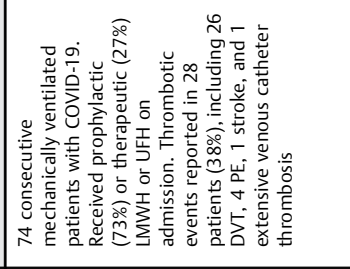 & 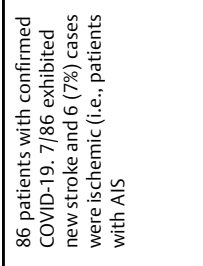 & 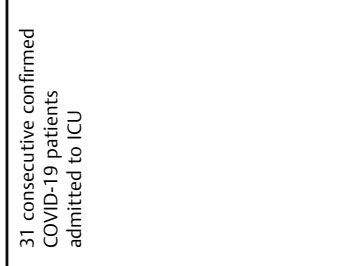 & 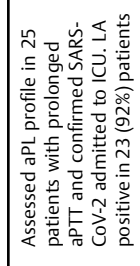 & 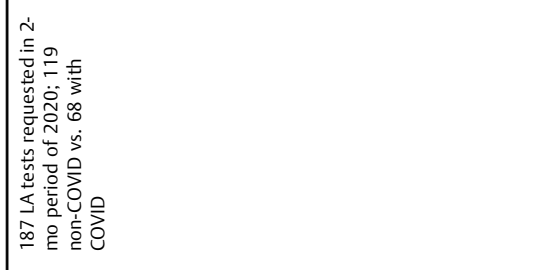 \\
\hline 苂 & 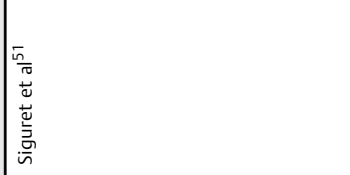 & 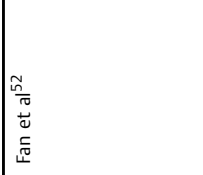 & 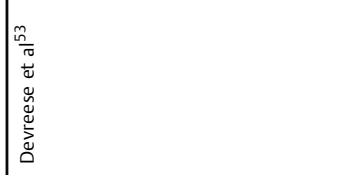 & 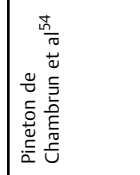 & 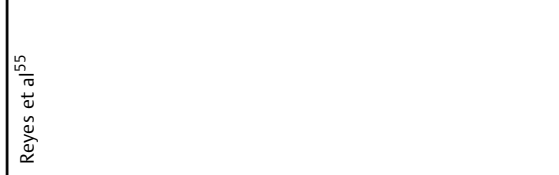 \\
\hline
\end{tabular}




\begin{tabular}{|c|c|c|c|c|}
\hline & 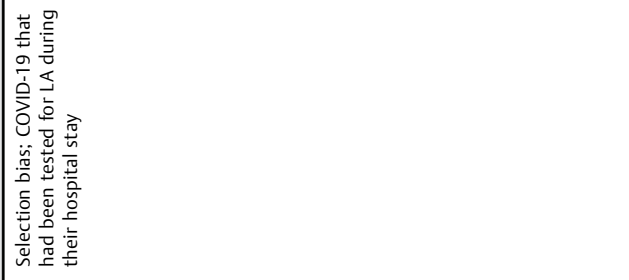 & & & \\
\hline 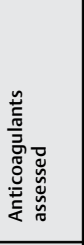 & 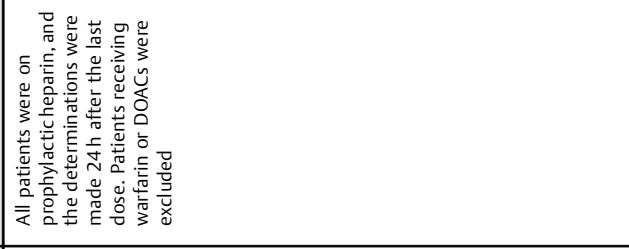 & $\frac{\alpha}{z}$ & 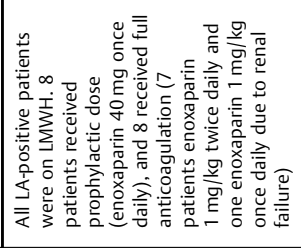 & 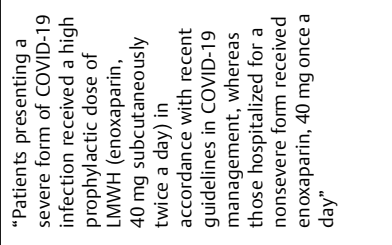 \\
\hline 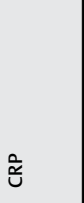 & 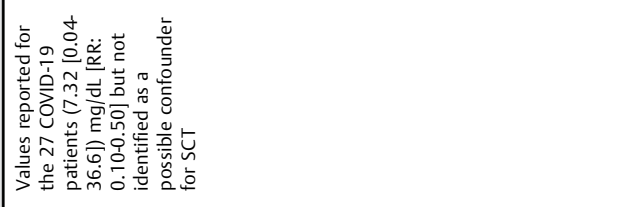 & . & 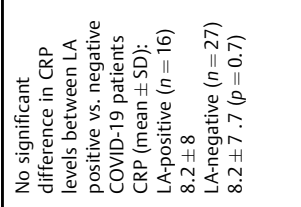 & 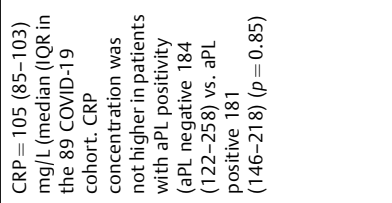 \\
\hline 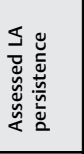 & $\frac{\alpha}{z}$ & $\frac{\alpha}{z}$ & $\frac{\alpha}{z}$ & $\frac{\mathscr{z}}{z}$ \\
\hline 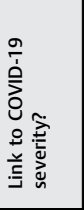 & 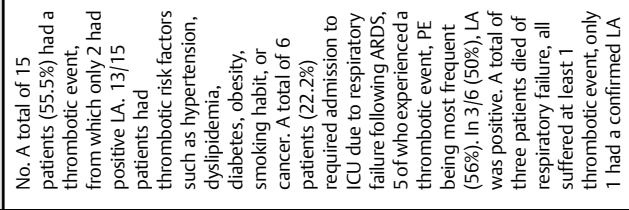 & $\frac{\approx}{2}$ & 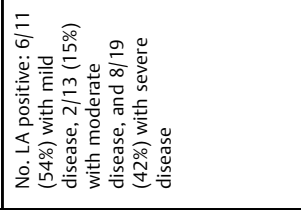 & 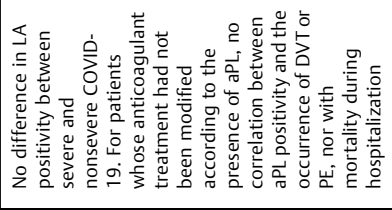 \\
\hline 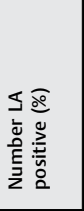 & 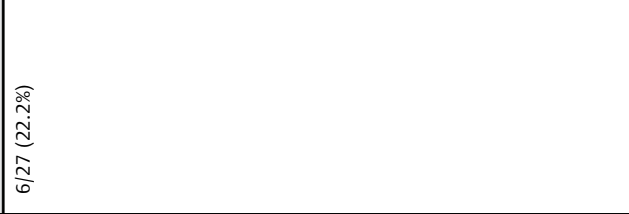 & $\frac{\alpha}{z}$ & 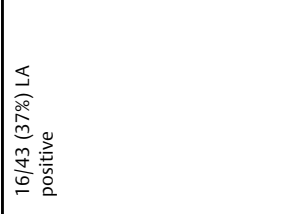 & 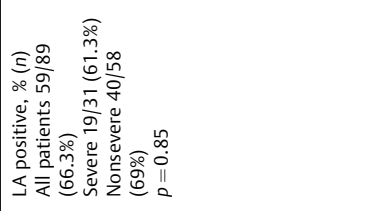 \\
\hline & 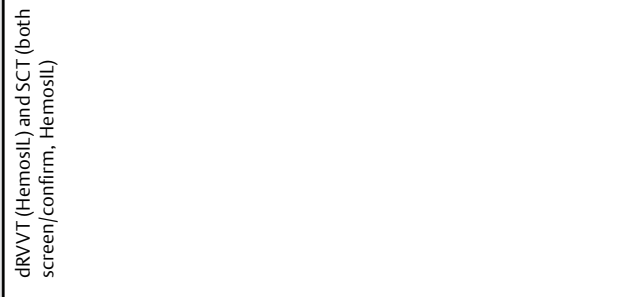 & $\stackrel{\alpha}{z}$ & 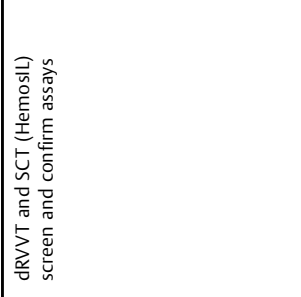 & 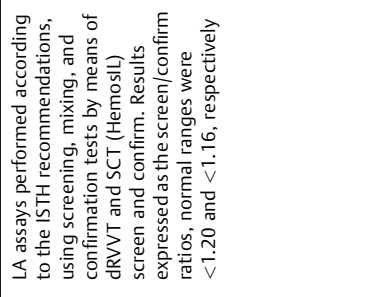 \\
\hline 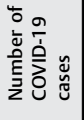 & $\lesssim$ & in & F & $\infty$ \\
\hline 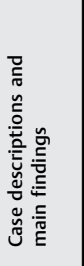 & 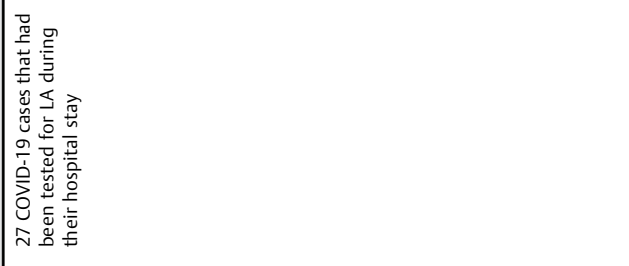 & 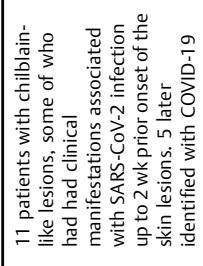 & 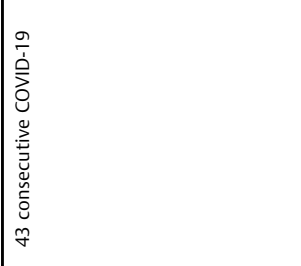 & 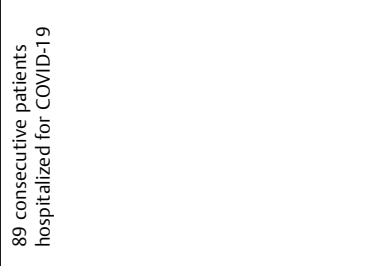 \\
\hline 苂 & 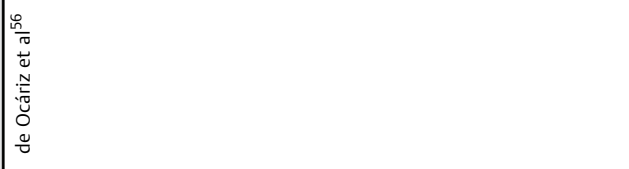 & 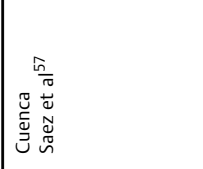 & 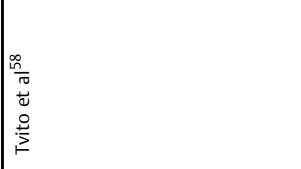 & 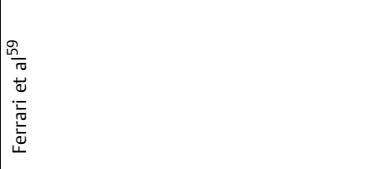 \\
\hline
\end{tabular}




\begin{tabular}{|c|c|c|c|c|c|}
\hline 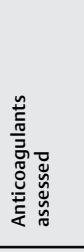 & 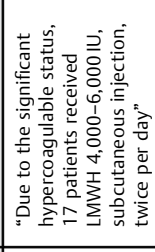 & 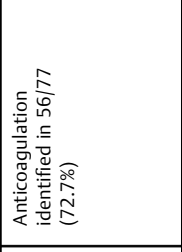 & 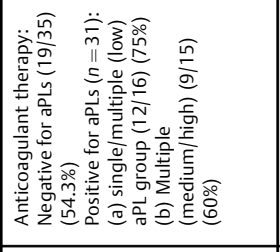 & 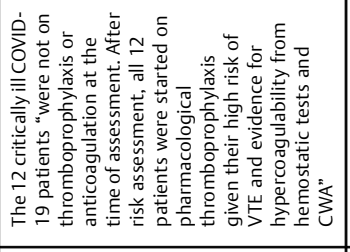 & 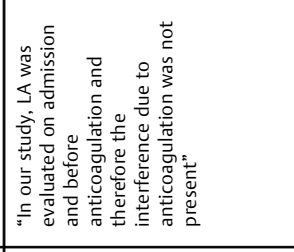 \\
\hline $\bar{\Xi}$ & $\stackrel{\alpha}{z}$ & 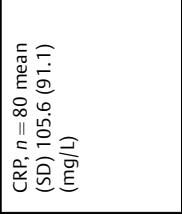 & 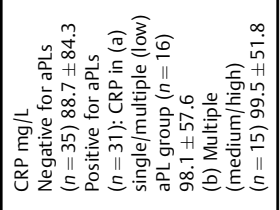 & $\frac{2}{2}$ & 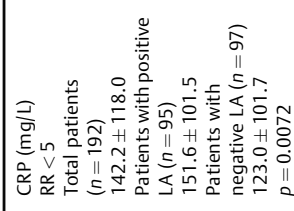 \\
\hline 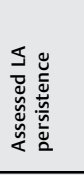 & 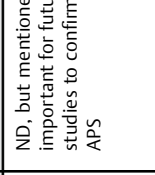 & $\stackrel{o}{z}$ & $\frac{o}{z}$ & $\stackrel{\sim}{z}$ & $\frac{x}{z}$ \\
\hline 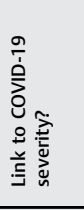 & 兰 & 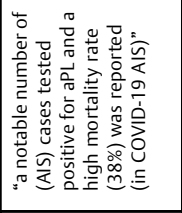 & 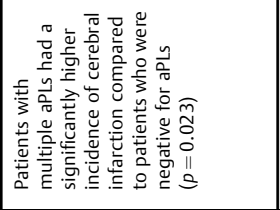 & 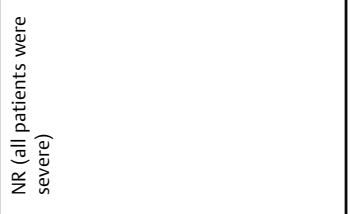 & 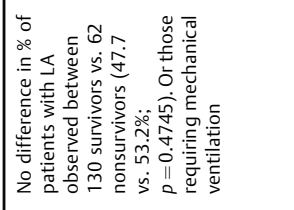 \\
\hline 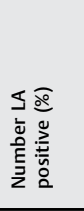 & 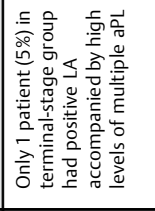 & 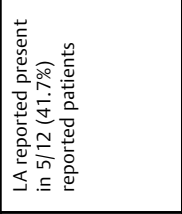 & 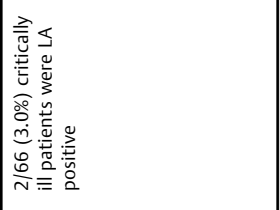 & 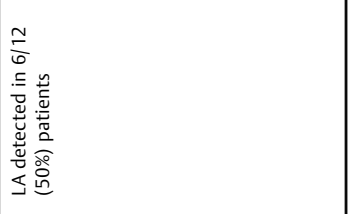 & 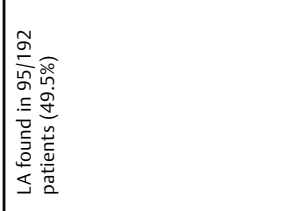 \\
\hline 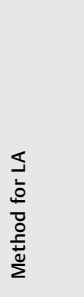 & 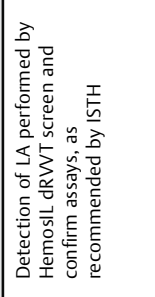 & 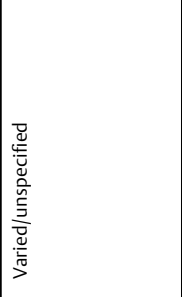 & 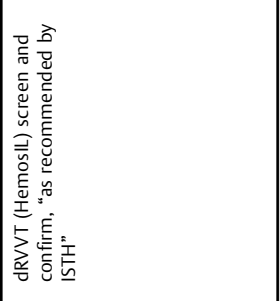 & 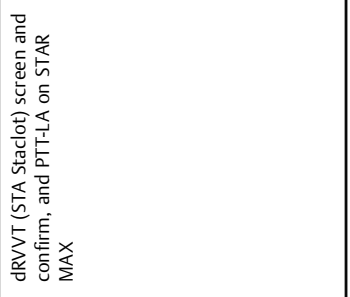 & 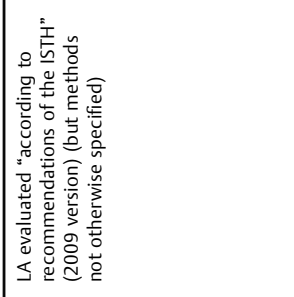 \\
\hline 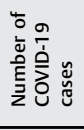 & ㅇ & $\stackrel{\stackrel{m}{m}}{\underline{n}}$ & I & $\simeq$ & $\stackrel{\Omega}{\Omega}$ \\
\hline 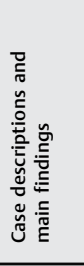 & 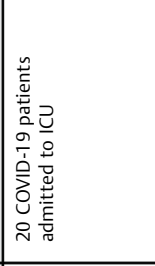 & 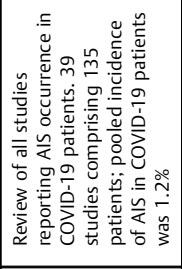 & 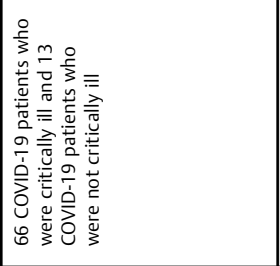 & 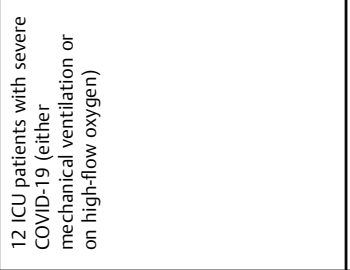 & 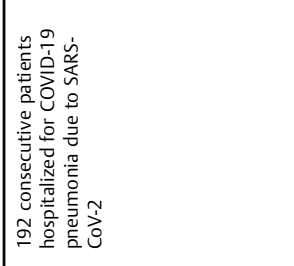 \\
\hline 巡 & 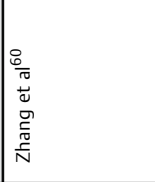 & 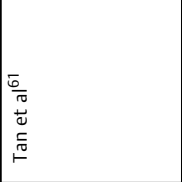 & 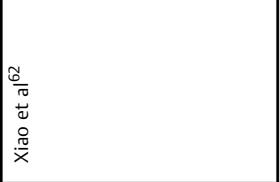 & 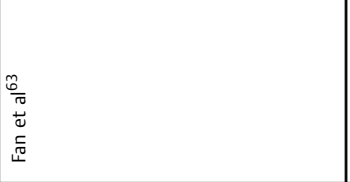 & 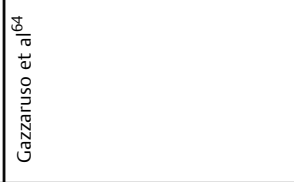 \\
\hline
\end{tabular}




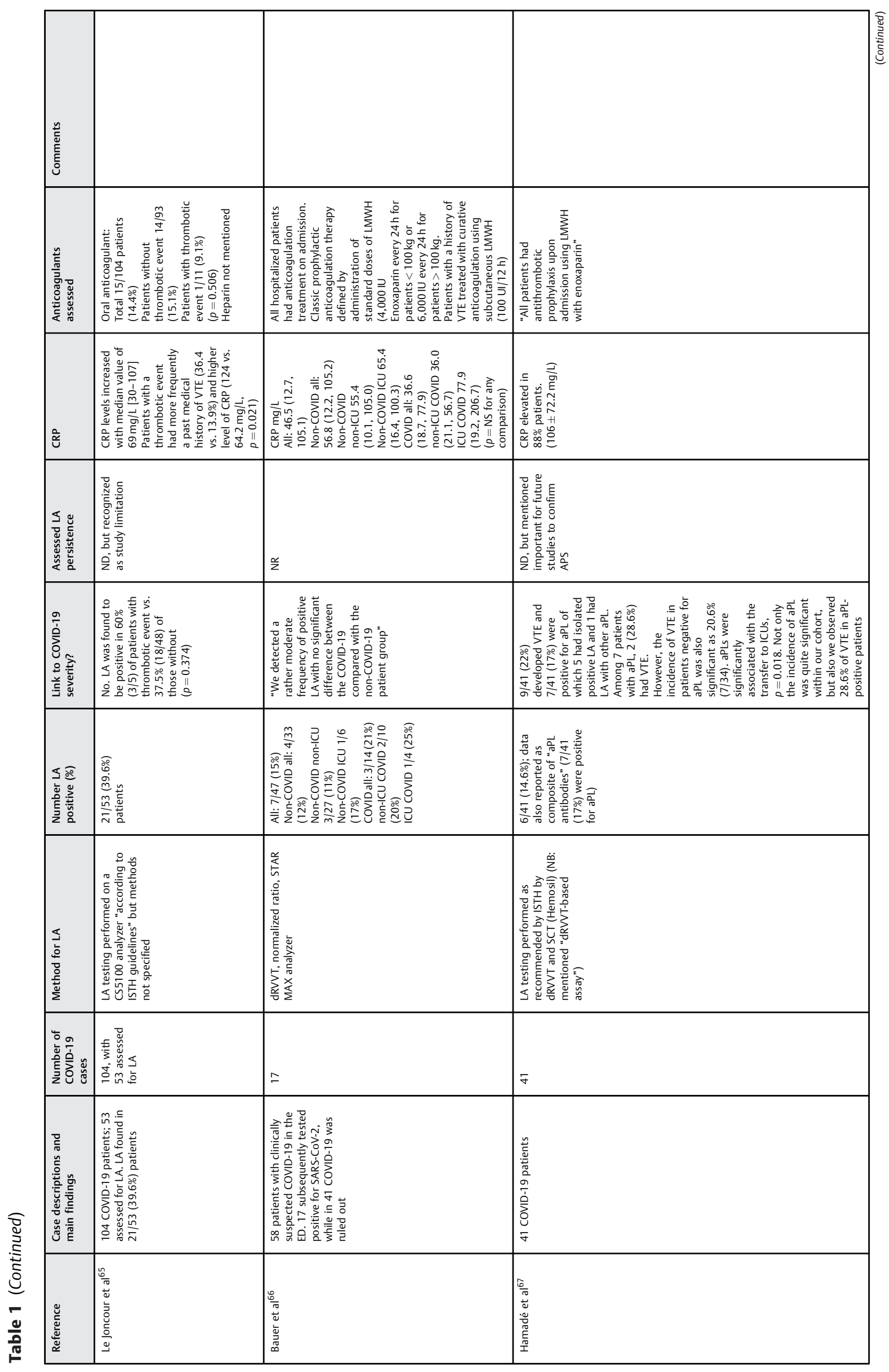



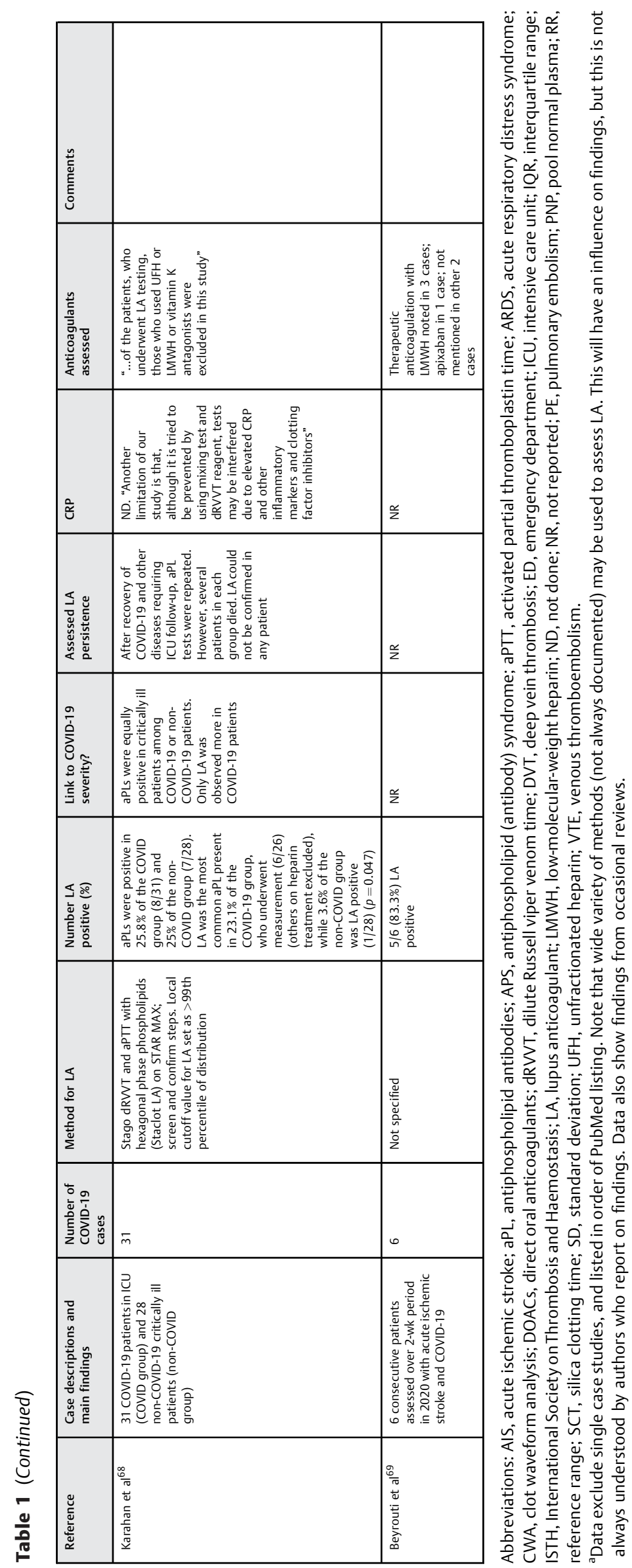
Zhang et al's report for aPL. ${ }^{17}$ Thus, there is likely to be additional selection bias in the literature where authors investigate LA (and other aPL). This bias can take two forms. First, researchers are more likely to publish positive findings than to publish negative findings. As an example, Tang 49 responding to a comment on one of his earlier articles indicated that "they had assessed LA in dozens of their COVID-19 patients and very few were positive." The second form of selection bias was apparent in several publications. Here, researchers actively looked for LA in select COVID-19 patient cohorts. This may include those who had raised aPTTs, or with clinical or laboratory suspicion of LA. In these studies, a relatively high level of LA was naturally identified in the studied COVID-19 population ${ }^{46,47}$. One can propose that this might be anticipated, and indeed findings of LA in patients investigated for prolonged aPTT or under clinical or laboratory suspicion of LA would be not unexpected, irrespective of the presence of COVID-19.

\section{C-Reactive Protein}

C-reactive protein(CRP) is well recognized by expertsin thefield to potentially generate false-positive LA findings, in particular using the aPTT. ${ }^{71,72}$ Indeed, if LA is identified only with the aPTT method, then CRPshould be excluded as a cause of false-positive LA. ${ }^{53,71,72}$ It is important to note that CRP is also highly elevated in patients with COVID-19, including those with reported LA. ${ }^{51,55,56,58,59,61,62,64-67}$ Interestingly, however, most researchers reporting on LA in COVID-19 did not mention CRP, nor report data on this biomarker. In some cases, these data may have possibly been reported elsewhere, and in other cases may not have been gathered or even considered. Of further interest, even when investigated or reported, CRP was not always contemplated by the researchers as a potential confounder for LA identification. Where reported, levels of CRP did not differ between COVID-19 cohorts found positive versus negative for LA, ${ }^{58,59,62}$ or else a statistically significant difference was reported. ${ }^{55,64}$ For example, Reyes et al $^{55}$ identified higher levels of CRP in patients testing positive for LA by dRVVT ( 14.4 vs. $7.5 \mathrm{mg} / \mathrm{dL} ; p<0.01$ ). They also reported that patients with thromboses did not have significantly higher CRP levels than those with no thromboses, and after adjusting for CRP, LA was found to be independently associated with thrombosis (odds ratio, 4.39; 95\% confidence interval: 1.45-14.57; $p=0.01)$. Gazzaruso et $\mathrm{a}^{64}$ also identified higher levels of CRP in patients with positive $\mathrm{LA}(n=95 ; 151.6 \pm 101.5 \mathrm{mg} / \mathrm{L})$ versus those with negative $\mathrm{LA}(n=97 ; 123.0 \pm 101.7 ; p=0.0072)$. Of course, none of this is the same as saying that the raised CRP in COVID-19 patients did not influence LA positivity, at least in a portion of "LA-positive" COVID-19 patients. However, it probably does suggest that CRP is not in itselfa majordriver of any false LA positivity in COVID-19 patients.

\section{Anticoagulants as a Confounder to LA Testing}

Similarly, many publications did not identify whether their COVID-19 cohorts were anticoagulated, or where patients were identified as anticoagulated, what anticoagulants were used for treatment. Some publications did identify the anticoagulants used for treatments, but failed to consider that these same anticoagulants could represent a confounder for LA testing. A few publications identified anticoagulants used for treatments and their possible presence as a confounder for LA testing.

In COVID-19, most patients would be under heparin therapy, with most under therapy with LMWH. Alternatively, some patients would be under DOAC therapy, and some under VKA therapy. Here, we need to reflect on treatment applied to prevent or treat thrombosis arising from COVID19 or its complications once admitted to hospital, which is likely to be LMWH(/UFH), versus patients who were already on an anticoagulant to treat or prevent thrombosis prior to contracting COVID-19, which then would more likely be a DOAC or a VKA. As mentioned previously, all anticoagulants affect LA testing, as summarized in - Table 2. Thus, the aPTT component of the LA test panel (or the SCT component, as used in some laboratories) would be sensitive to all the anticoagulants (VKAs, all heparins, DOACs). Mitigation of any anticoagulant effect on aPTT or SCT, as used for LA testing, is difficult, as also outlined in - Table 2. Note that the aPTT in particular is also used to monitor UFH therapy, and thus may be purposely designed to be particularly sensitive to UFH. Nonetheless, the SCT would also be very sensitive to UFH. Although it is generally considered that the aPTT is not highly sensitive to LMWH, given the predominant anti-Xa activity (as opposed to predominant anti-Ila activity of UFH), both aPTT and SCT would have some sensitivity to LMWH, according to the concentration present. The dRVVT would be sensitive to VKAs and DOACs, and less sensitive to UFH/LMWH because most commercial reagents contain heparin neutralizers, quenching the heparin activity when within the therapeutic range, and generally up to $1 \mathrm{U} / \mathrm{mL}$ heparin. Nevertheless, higher concentrations will affect the dRVVT, which, in the absence of heparin neutralization, becomes very sensitive to heparin.

Some researchers had different strategies for mitigating heparin interference. For example, Devreese et al $^{53}$ surmised that "applying the three-step procedure, UFH does not result in false-positive LA, whereas enoxaparin (LMWH) causes false-positive LA at supratherapeutic anti-Xa activity levels that exceed the heparin neutralizing capabilities of the reagents. $^{73,74 "}$

For VKAs, the only solution is to either avoid testing or perform mixing studies with normal plasma ${ }^{25}$ to correct for the VKA-induced factor deficiency (factors II, VII, IX, X), although this is no longer recommended by the ISTH Scientific and Standardization Committee (SSC) on LA. ${ }^{23}$ This would apply to all the LA assays (dRVVT, aPTT, SCT). For heparin, mixing would reduce the effect on the aPTT and SCT, and possibly correct any effect on the dRVVT, should the dilution then lead to a heparin level within a therapeutic range (or generally $<1 \mathrm{U} / \mathrm{mL}$ ). For DOACs, one could use DOAC neutralizers such as DOAC Stop or DOAC Remove, ${ }^{34}$ although this in itself may have an unexpected effect on LA detection. Irrespective, laboratories would need to apply such 
Table 2 Effects of anticoagulants on main assays used to investigate LA

\begin{tabular}{|c|c|c|c|c|}
\hline Anticoagulant & $\begin{array}{l}\text { Affects } \\
\text { aPTT }\end{array}$ & $\begin{array}{l}\text { Affects } \\
\text { SCT }\end{array}$ & Affects dRVVT & Strategies for mitigating effects \\
\hline $\begin{array}{l}\text { VKAs } \\
\text { (e.g., warfarin) }\end{array}$ & ++ & ++ & +++ & $\begin{array}{l}\text { 1. Avoid testing while on therapy } \\
\text { 2. Use mixing with normal plasma to normalize } \\
\text { factor levels (but may still lead to false-positive or } \\
\text {-negative LA, and no longer recommended by } \\
\text { the ISTH }{ }^{23,74} \text { ) }\end{array}$ \\
\hline UFH & +++ & ++++ & $\begin{array}{l}- \text { (therapeutic level) } \\
\text { to }+++ \text { (supratherapeutic } \\
\text { level) }\end{array}$ & $\begin{array}{l}\text { 1. Avoid testing while on therapy } \\
\text { 2. Use heparin neutralizer (present in dRVVT } \\
\text { reagent)-but won't eliminate all heparin if } \\
\text { supratherapeutic } \\
\text { 3. Use “3-step procedure" for LA testing } 23,74\end{array}$ \\
\hline LMWH & + to ++ & ++ & $\begin{array}{l}- \text { (therapeutic level) } \\
\text { to }+++ \text { (supratherapeutic } \\
\text { level) }\end{array}$ & $\begin{array}{l}\text { 1. Avoid testing while on therapy } \\
\text { 2. Use heparin neutralizer (present in dRVVT } \\
\text { reagent)-but won't eliminate all heparin if } \\
\text { supratherapeutic } \\
\text { 3. Test at trough (prior to next dose) }\end{array}$ \\
\hline DOACs & + to +++ & + to +++ & + to +++ & $\begin{array}{l}\text { 1. Avoid testing while on therapy } \\
\text { 2. Use DOAC neutralizer (not present in dRVVT } \\
\text { reagents; purchased separately) }\end{array}$ \\
\hline
\end{tabular}

Abbreviations: aPTT, activated partial thromboplastin time; dRVVT, dilute Russell viper venom time; DOACs, direct oral anticoagulants; LA, lupus anticoagulant; LMWH, low-molecular-weight heparin; ISTH, International Society on Thrombosis and Haemostasis ${ }^{23,74}$; SCT, silica clotting time (a form of aPTT); UFH, unfractionated heparin; VKA, vitamin $\mathrm{K}$ antagonist.

strategies to mitigate the effect of any anticoagulant and ensure appropriate detection of LA. Thus, laboratories would need to be aware of any anticoagulant effect on the potential for false-positive identification of LA, and also then attempt to mitigate for said effect prior to identification of LA, otherwise a false positive can ensue.

Furthermore, anticoagulants, especially DOACs, but potentially also heparin, may have a different effect on the screen versus confirm assays, and this will affect any resultant ratio value. It is often the ratio value that is used for identification versus exclusion of LA, which for dRVVT screen/confirm is often a cutoff value of around $1.2 .^{75}$ Thus, values below would normally exclude LA, whereas values above would infer LA positivity. Complicating this further, the best approach would be a normalized ratio, which to some extent could mitigate the differential effect on screen versus confirm reagents, but it is not clear if this strategy is used in all laboratories reporting LA in COVID-19.

-Figs. 2 and $\mathbf{3}$ show some examples of these concepts applied in practice, respectively, for LMWH and one of the DOACs, rivaroxaban. Note the differential effect of LMWH on the aPTT reagents used as the screen and confirm component (-Fig.2). Similarly, note the differential effect of rivaroxaban on the dRVVT reagents used as the screen and confirm component (-Fig. 3). For this aPTT example, the greater effect was observed on the confirm component than on the screen, and thus a false-positive LA by aPTT in a patient using LMWH seems less likely. However, other aPTT reagent pairs may show the reverse pattern. For the dRVVT example, the interference effect is greater on the screen than the confirm component, and thus an LA ratio above 1.2 is certainly possible, leading to possible false-positive LA by dRVVT.
In summary, then, it is likely that at least some of the positive LA findings reported in the literature reflect false positives due to anticoagulant effects that have not been appropriately accounted for by some researchers.

\section{Persistence of LA Positivity versus Transient Positivity}

To identify LA or other aPL as a specific feature of an autoimmune disorder such as APS, one has to prove the persistence of that positivity, generally by repeating the test(s) on a second sample some 12 weeks after the first positive test result. ${ }^{1,2,23}$ Again, most researchers reporting on LA positivity in COVID-19 either did not mention this or did not undertake repeated testing. Thus, persistence of LA positivity was not evaluated in most studies, and hence not proven. In the few studies that did attempt to look at persistence, most cases initially positive for LA then became negative for $\mathrm{LA}^{53}$ or else repeat testing was complicated by the ongoing patient morbidity or their death. ${ }^{68}$ Thus, it seems that any LA positivity that may be identified in COVID-19 patients is mostly transient.

\section{Transient aPLs Are a Common Feature of Severe Viral Infections}

It is well known among those looking after sick patients with various viral infections that aPL may transiently appear in a range of conditions. ${ }^{76,77}$ It may be possible to separate groups of patients and aPL profiles. For example, in one meta-analysis, Abdel-Wahab et al $^{77}$ reported that three different groups of patients could be identified: "group 1 
A.

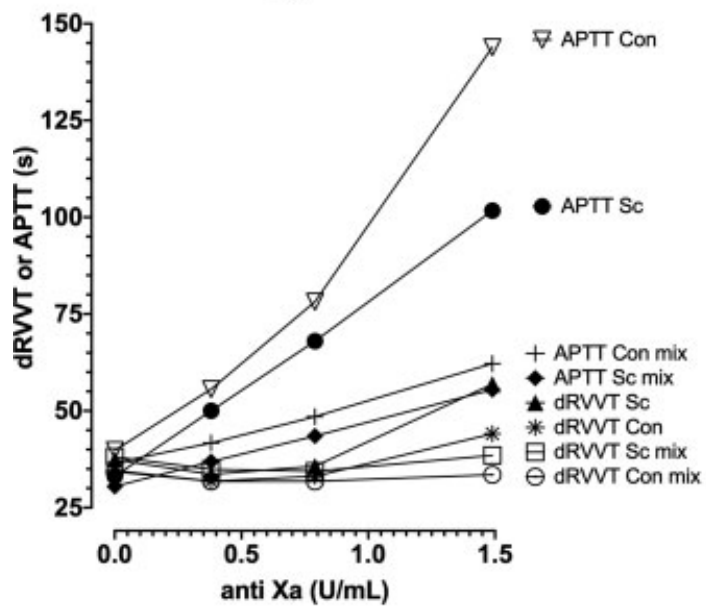

B.

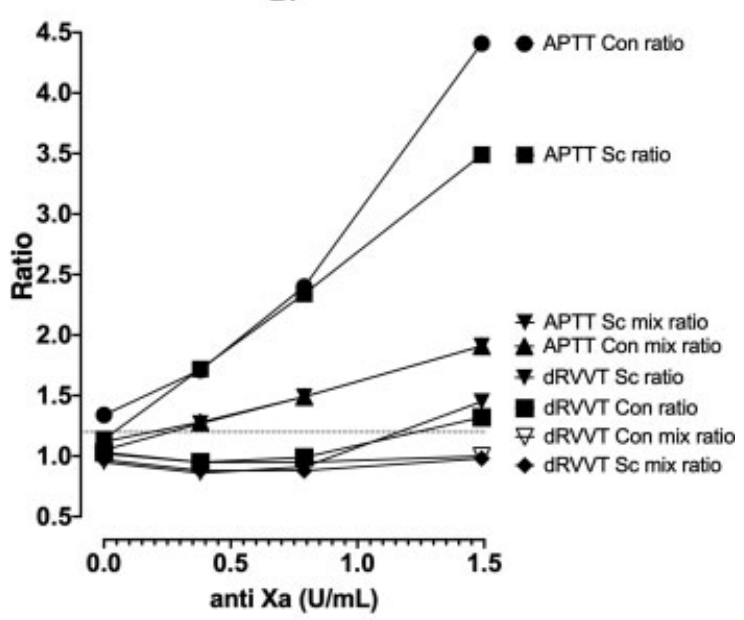

C.

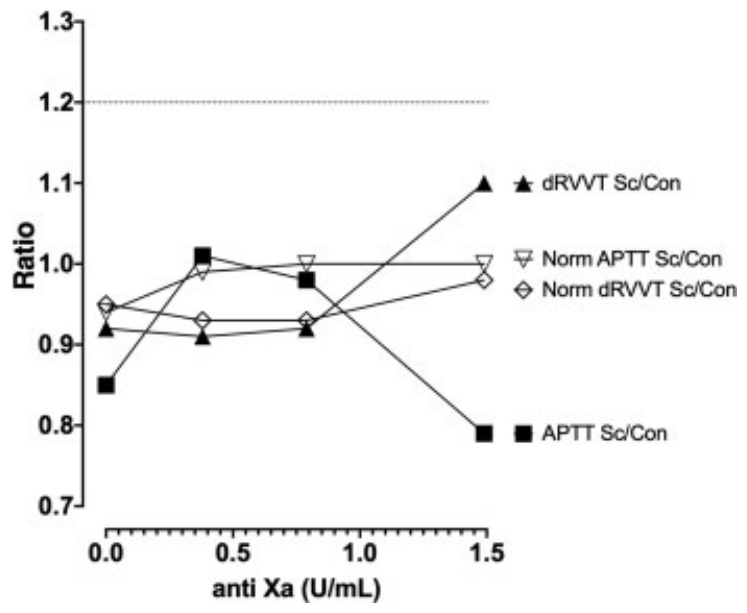

Fig. 2 The effect of low-molecular-weight heparin (LMWH) on some common lupus anticoagulant (LA) tests. Normal plasma was spiked with increasing concentrations of enoxaparin, ranging from 0 to 1.5 $\mathrm{U} / \mathrm{mL}$, and then tests for aPTT (activated partial thromboplastin time) and dRVVT (dilute Russell viper venom time) were performed. While it is recognized that LMWH spiked samples do not behave exactly the same as ex vivo samples, this exercise is useful to show some anomalies in LA test results. (A) Effect on aPTT and dRVVT clotting times: (i) note differential effect on aPTT screen (Sc, Siemens Actin FSL included patients who fulfilled the criteria for definitive APS (24.6\%), group 2 included patients who developed transient aPL with thromboembolic phenomena (43.7\%), and group 3 included patients who developed transient aPL without thromboembolic events (31.7\%). Thus, secondary cases of APS due to viral infections have been reported. ${ }^{78}$ Secondary cases of APS due to infectious agents potentially evolving into CAPS have also been reported and include infections from hepatitis C virus, herpes zoster, as well as bacteria, fungi, parasites, and acute $\mathrm{Q}$ fever. ${ }^{79}$ The induction of molecular mimicry that leads to production of anti-beta2 glycoprotein I (aß2GPI) autoantibodies has been proposed as putative cause of secondary APS and CAPS. ${ }^{80,81}$

Thus, the finding of LA positivity in COVID-19 is not unique to COVID-19. To our knowledge, there is no evidence available on comparative infections with other viral agents to identify if the situation in COVID-19 in regard to aPL and LA positivity is worse or greater than that of other severe viral infections. In part, it is also likely that other viral diseases have not been as extensively studied as COVID-19.

reagent; LA sensitive) vs. that on aPTT confirm assay (Con, Siemens Actin FS reagent; LA insensitive due to added phospholipid). For this reagent pair, LMWH affects the confirm assay (FS) more than the screen assay (FSL); (ii) a reduced effect is seen on the aPTT assays when performed as mixes with normal plasma; here, the essential consequence is a reduction in LMWH concentration; however, the effect is still greater on the confirm assay (FS) than the screen assay (FSL). Although for the aPTT pair evaluated here, the effect was greater on the confirm assay than on screen, not all aPTT reagent pairs may show this pattern, and the reverse (greater effect on the screen than confirm) is also possible. (iii) A reduced effect is seen with the dRVVT assay, since the reagents contain a heparin neutralizer. Essentially, an effect is seen only for the high LMWH concentration of 1.5 $\mathrm{U} / \mathrm{mL}$, and is not seen when the RVVT is performed as a mix test, since the resultant diluted LMWH is able to then be neutralized by the reagent. Nevertheless, the $\mathrm{LMWH}$ effect is greater on the screen reagent than the confirm reagent. (B) Effect on aPTT and dRVVT ratios. Data from $(A)$ plotted as assay ratios (i.e., aPTT and dRVVT clotting times in (A) in comparison with normal plasma test times). All aPTT ratios, being the screen and confirm, and also when performed as a mix with normal plasma, are $>1.2$. Although this in itself cannot be used to identify LA, it may be used to decide on further evaluation for $\mathrm{LA}$ by additional testing. Only the dRVVT ratios for the highest LMWH concentration are above 1.2, and only when performed as neat plasma (not when performed as a mix with normal plasma) (due to the presence of heparin neutralizer in the reagents). (C) Effect on aPTT and dRVVT final ratios including normalized ratios. Data from ( $A$ and B) plotting screen/confirm ratios including normalized ratios, which essentially normalize the test results by taking into account clotting times obtained with normal plasma. The normalized ratios are similar and close to 1.0 irrespective of the LMWH concentration. Normalized ratios are recommended for use by the LA guidelines. In contrast, the nonnormalized ratios vary according to LMWH concentration. In this example, the highest LMWH concentration has differential effects on screen vs confirm reagents, and also differential effects on aPTT vs. dRVVT. Thus, for aPTT, the non-normalized ratio is $<1.0$, and for the dRVVT the non-normalized ratio is $>1.0$. It is possible that for some aPTT and dRVVT reagent pairs, the differential could be so great as to create ratios $>1.2$, or at least greater than a laboratory determined cut-off value, and thus increase the potential for false-positive LA, should nonnormalized ratios be utilized by a laboratory for assessing the presence of LA. 


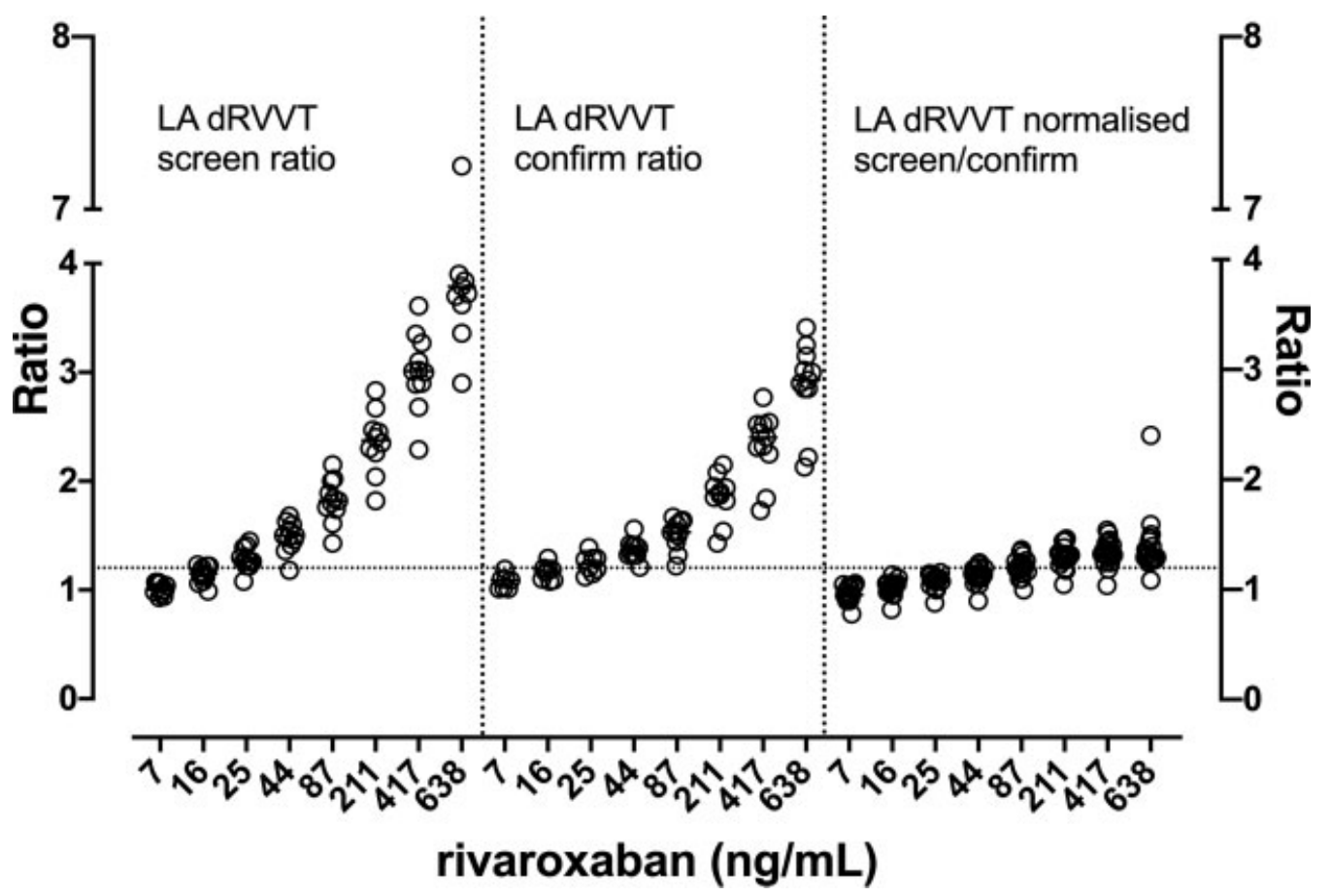

Fig. 3 The effect of rivaroxaban on lupus anticoagulant (LA) testing by dilute Russell viper venom time (dRVVT). Increasing concentrations of rivaroxaban ( $x$-axis) have a corresponding effect on both dRVVT screen (left portion of figure) and dRVVT confirm (middle portion of figure). However, the effect is greater on dRVVT screen than on dRVVT confirm. Thus, dRVVT screen/confirm ratios (even if normalization) can exceed 1.2 , or the cutoff used in laboratories to determine LA, and therefore lead to a false conclusion of LA. This occurs at concentrations of rivaroxaban seen in patients on rivaroxaban therapy.

\section{Does LA Positivity in COVID-19 Reflect a Risk Factor for Thrombosis?}

Only a few studies investigated whether LA positivity inferred additional thrombotic risk. Few studies identified a statistical difference in thrombotic risk for LA-positive versus LA-negative patients, ${ }^{52,55}$ whereas most did not. $^{50,51,56,58,59,64-67}$ There are many potential confounders in this evaluation, and it is unclear if these confounders were considered in all published comparisons. Thus, transient aPL (or LA) positivity may develop in the sickest patients, who will then be most at risk of thrombosis, and therefore LA may just reflect an association with, rather than be responsible for, the pathophysiological events. Irrespective, whether LA positivity in COVID-19 truly reflects an additional risk factor for thrombosis remains currently unresolved.

\section{General Discussion}

Taking all this information into consideration, we would propose that LA positivity is a feature of COVID-19, at least in some patients, and potentially those who are the sickest or have the most severe infection. However, we also believe that a proportion of cases identified in the literature as being LA positive reflect false positives, and potentially due to confounding by preanalytical issues, such as patients being on anticoagulants at the time of blood sampling, as well as analytical issues, which are not always easy to identify from the published studies. All anticoagulants affect LA testing, and it is unlikely that all studies took these anticoagulants into account in regard when performing tests and reporting findings, or else perhaps assumed no effect because patients were on therapeutic LMWH therapy. Such assumptions may not be valid, as shown in - Fig. 2, depending on which assays are performed, and how they are performed and reported. Mitigation of DOAC effects would be difficult, and although achievable using DOAC neutralizers, ${ }^{34,74,82}$ may again not have been recognized by researchers reporting their results.

Repeat testing for persistence of LA was rarely performed or reported, and where reported suggested a transient nature of the identified "LA." Such transient LA does not identify an autoimmune disease in the classic sense of APS. ${ }^{1,2}$ Such transient aPLs are also commonly observed in other viral infections, ${ }^{76,77}$ and thus do not seem to be unique to COVID-19. There are also questions remaining over the "additional" thrombotic risk imposed by the LA identified in COVID-19 in these studies, as transient aPLs developed from viral infections are often not associated with thrombosis.

\section{Conclusion}

Larger and better studies are needed to address the residual question regarding the true frequency of LA in COVID-19, and whether these laboratory-detected LA would actually contribute to enhance the thrombotic risk in COVID-19. Nevertheless, we believe that some good-quality studies have already been published, and these should likely guide 
opinion. These studies are those that reported on LA cognizant of the potential confounders, including CRP and anticoagulant therapy, and which also looked at persistence of antibodies. However, they were in the minority of published studies. All this is not to say that APS cannot develop in patients with COVID-19. As already mentioned, there are certainly similarities between the worst presentation of APS, namely CAPS, and what occurs in the sickest patients with COVID-19. But there are also some notable differences, including general lack of high titer aPL, lack of persistence for LA and other aPL, and unclear relationship between the detected aPL/LA and COVID-19-associated coagulopathy.

\section{Conflict of Interest}

None declared.

\section{Acknowledgments}

Some of the data shown in - Figs. 2 and $\mathbf{3}$ derive from the RCPA Haematology QAP (Royal College of Pathologists of Australasia Quality Assurance Program) or material supplied by them. We thank current and past staff of the RCPAQAP, including Roslyn Bonar, Sandya Arunachalam, and Elysse Dean. We also thank Ronny Vong and Elizabeth Duncan. The opinions expressed in this review are those of the authors, and do not necessarily reflect the opinions of their respective employers, NSW Health Pathology, The Heart Institute, Cincinnati Children's Hospital Medical Center, or the University of Verona.

\section{References}

1 Miyakis S, Lockshin MD, Atsumi T, et al. International consensus statement on an update of the classification criteria for definite antiphospholipid syndrome (APS). J Thromb Haemost 2006;4 (02):295-306

2 Devreese KMJ, Ortel TL, Pengo V, de Laat BSubcommittee on Lupus Anticoagulant/Antiphospholipid Antibodies. Laboratory criteria for antiphospholipid syndrome: communication from the SSC of the ISTH. J Thromb Haemost 2018;16(04):809-813

3 Molhoek JE, de Groot PG, Urbanus RT. The lupus anticoagulant paradox. Semin Thromb Hemost 2018;44(05):445-452

4 Favaloro EJ, Wong RCW. Antiphospholipid antibody testing for the antiphospholipid syndrome: a synopsis of challenges and recent guidelines. Pathology 2014;46(06):481-495

5 Galli M, Luciani D, Bertolini G, Barbui T. Lupus anticoagulants are stronger risk factors for thrombosis than anticardiolipin antibodies in the antiphospholipid syndrome: a systematic review of the literature. Blood 2003;101(05):1827-1832

6 Lippi G, Sanchis-Gomar F, Favaloro EJ, Lavie CJ, Henry BM. Coronavirus disease 2019-associated coagulopathy. Mayo Clin Proc 2021;96(01):203-217

7 Di Minno A, Ambrosino P, Calcaterra I, Di Minno MND. COVID-19 and venous thromboembolism: a meta-analysis of literature studies. Semin Thromb Hemost 2020;46(07):763-771

8 Jenner WJ, Kanji R, Mirsadraee S, et al. Thrombotic complications in 2928 patients with COVID-19 treated in intensive care: a systematic review. J Thromb Thrombolysis 2021;51(03):595-607

9 Uaprasert N, Moonla C, Sosothikul D, Rojnuckarin P, Chiasakul T. Systemic coagulopathy in hospitalized patients with coronavirus disease 2019: a systematic review and meta-analysis. Clin Appl Thromb Hemost 2021;27:1076029620987629

10 Carsana L, Sonzogni A, Nasr A, et al. Pulmonary post-mortem findings in a series of COVID-19 cases from northern Italy: a two- centre descriptive study. Lancet Infect Dis 2020;20(10): $1135-1140$

11 Wichmann D, Sperhake JP, Lütgehetmann M, et al. Autopsy findings and venous thromboembolism in patients with COVID19. Ann Intern Med 2020;173(04):268-277

12 Bradley BT, Maioli H, Johnston R, et al. Histopathology and ultrastructural findings of fatal COVID-19 infections in Washington State: a case series. Lancet 2020;396(10247):320-332

13 Varga Z, Flammer AJ, Steiger P, et al. Endothelial cell infection and endotheliitis in COVID-19. Lancet 2020;395(10234):1417-1418

14 Mendoza-Pinto C, Escárcega RO, García-Carrasco M, Bailey DJO, Gálvez-Romero JL, Cervera R. Viral infections and their relationship with catastrophic antiphospholipid syndrome: a possible pathogenic mechanism of severe COVID-19 thrombotic complications. J Intern Med 2020;288(06):737-739

15 El Hasbani G, Taher AT, Jawad A, Uthman I. COVID-19, antiphospholipid antibodies, and catastrophic antiphospholipid syndrome: a possible association? Clin Med Insights Arthritis Musculoskelet Disord 2020;13:1179544120978667

16 Previtali G, Seghezzi M, Moioli V, et al. The pathogenesis of thromboembolic disease in covid-19 patients: Could be a catastrophic antiphospholipid syndrome? Thromb Res 2020; 194:192-194

17 Zhang Y, Xiao M, Zhang S, et al. Coagulopathy and antiphospholipid antibodies in patients with Covid-19. N Engl J Med 2020;382 (17): $\mathrm{e} 38$

18 Metjian A, Lim W. ASH evidence-based guidelines: should asymptomatic patients with antiphospholipid antibodies receive primary prophylaxis to prevent thrombosis? Hematology (Am Soc Hematol Educ Program) 2009:247-249

19 Mustonen P, Lehtonen KV, Javela K, Puurunen M. Persistent antiphospholipid antibody (aPL) in asymptomatic carriers as a risk factor for future thrombotic events: a nationwide prospective study. Lupus 2014;23(14):1468-1476

20 Pengo V, Ruffatti A, Legnani C, et al. Incidence of a first thromboembolic event in asymptomatic carriers of high-risk antiphospholipid antibody profile: a multicenter prospective study. Blood 2011;118(17):4714-4718

21 Yelnik CM, Urbanski G, Drumez E, et al. Persistent triple antiphospholipid antibody positivity as a strong risk factor of first thrombosis, in a long-term follow-up study of patients without history of thrombosis or obstetrical morbidity. Lupus 2017;26 (02):163-169

22 Larsen JB, Hvas AM. Predictive value of whole blood and plasma coagulation tests for intra- and postoperative bleeding risk: a systematic review. Semin Thromb Hemost 2017;43(07):772-805

23 Devreese KMJ, de Groot PG, de Laat B, et al. Guidance from the Scientific and Standardization Committee for lupus anticoagulant/antiphospholipid antibodies of the International Society on Thrombosis and Haemostasis: update of the guidelines for lupus anticoagulant detection and interpretation. J Thromb Haemost 2020;18(11):2828-2839

24 Exner T, Triplett DA, Taberner D, Machin SJSSC Subcommittee for the Standardization of Lupus Anticoagulants. Guidelines for testing and revised criteria for lupus anticoagulants. Thromb Haemost 1991;65(03):320-322

25 Pengo V, Tripodi A, Reber G, et al; Subcommittee on Lupus Anticoagulant/Antiphospholipid Antibody of the Scientific and Standardisation Committee of the International Society on Thrombosis and Haemostasis. Update of the guidelines for lupus anticoagulant detection. J Thromb Haemost 2009;7(10): $1737-1740$

26 Keeling D, Mackie I, Moore GW, Greer IA, Greaves MBritish Committee for Standards in Haematology. Guidelines on the investigation and management of antiphospholipid syndrome. Br J Haematol 2012;157(01):47-58

27 Ledford-Kraemer M, Moore GW, Bottenus R, et al. Clinical and Laboratory Standards Institute (CLSI). Laboratory Testing for the 
Lupus Anticoagulant; Approved Guideline. CLSI document H60-A. Wayne, PA: CLSI; 2014

28 Tripodi A, Chantarangkul V. Lupus anticoagulant testing: activated partial thromboplastin time (APTT) and silica clotting time (SCT). Methods Mol Biol 2017;1646:177-183

29 Pengo V, Bison E, Banzato A, Zoppellaro G, Jose SP, Denas G. Lupus anticoagulant testing: diluted Russell viper venom time (dRVVT). Methods Mol Biol 2017;1646:169-176

30 Favaloro EJ, Lippi G. Interference of direct oral anticoagulants in haemostasis assays: high potential for diagnostic false positives and false negatives. Blood Transfus 2017;15(06):491-494

31 Favaloro EJ, Mohammed S, Curnow J, Pasalic L. Laboratory testing for lupus anticoagulant (LA) in patients taking direct oral anticoagulants (DOACs): potential for false positives and false negatives. Pathology 2019;51(03):292-300

32 Gosselin RC, Adcock DM, Bates SM, et al. International Council for Standardization in Haematology (ICSH) recommendations for laboratory measurement of direct oral anticoagulants. Thromb Haemost 2018;118(03):437-450

33 Favaloro EJ, Pasalic L, Curnow J, Lippi G. Laboratory monitoring or measurement of direct oral anticoagulants (DOACs): advantages, limitations and future challenges. Curr Drug Metab 2017;18(07): 598-608

34 Exner T, Rigano J, Favaloro EJ. The effect of DOACs on laboratory tests and their removal by activated carbon to limit interference in functional assays. Int J Lab Hematol 2020;42(Suppl 1):41-48

35 COVID-19 Dashboard by the Center for Systems Science and Engineering (CSSE) at Johns Hopkins University. Accessed March 16, 2021 at: https://www.arcgis.com/apps/opsdashboard/index. html\#/bda7594740fd40299423467b48e9ecf6

36 Favaloro EJ, Lippi G. Recommendations for minimal laboratory testing panels in patients with COVID-19: potential for prognostic monitoring. Semin Thromb Hemost 2020;46(03):379-382

37 Christensen B, Favaloro EJ, Lippi G, Van Cott EM. Hematology laboratory abnormalities in patients with coronavirus disease 2019 (COVID-19). Semin Thromb Hemost 2020;46(07):845849

38 Levi M, Thachil J. Coronavirus disease 2019 coagulopathy: disseminated intravascular coagulation and thrombotic microangiopathy-either, neither, or both. Semin Thromb Hemost 2020;46 (07):781-784

39 Thachil J, Srivastava A. SARS-2 coronavirus-associated hemostatic lung abnormality in COVID-19: Is it pulmonary thrombosis or pulmonary embolism? Semin Thromb Hemost 2020;46(07): 777-780

40 Schulman S. Coronavirus disease 2019, prothrombotic factors, and venous thromboembolism. Semin Thromb Hemost 2020;46 (07):772-776

41 Kwaan HC. Coronavirus disease 2019: the role of the fibrinolytic system from transmission to organ injury and sequelae. Semin Thromb Hemost 2020;46(07):841-844

42 Larsen JB, Pasalic L, Hvas AM. Platelets in coronavirus disease 2019. Semin Thromb Hemost 2020;46(07):823-825

43 Favaloro EJ, Henry BM, Lippi G. Increased VWF and decreased ADAMTS13 in COVID-19: creating a milieu for (micro)thrombosis? Semin Thromb Hemost 2021;19(02):513-521

44 Yasri S, Wiwanitkit V. COVID-19, antiphospholipid syndrome and thrombosis. Clin Appl Thromb Hemost 2020;26:107602962093 1927

45 Andina D, Noguera-Morel L, Bascuas-Arribas M, et al. Chilblains in children in the setting of COVID-19 pandemic. Pediatr Dermatol 2020;37(03):406-411

46 Helms J, Tacquard C, Severac F, et al; CRICS TRIGGERSEP Group (Clinical Research in Intensive Care and Sepsis Trial Group for Global Evaluation and Research in Sepsis) High risk of thrombosis in patients with severe SARS-CoV-2 infection: a multicenter prospective cohort study. Intensive Care Med 2020;46(06): 1089-1098
47 Bowles L, Platton S, Yartey N, et al. Lupus anticoagulant and abnormal coagulation tests in patients with Covid-19. N Engl J Med 2020;383(03):288-290

48 Harzallah I, Debliquis A, Drénou B. Lupus anticoagulant is frequent in patients with Covid-19. J Thromb Haemost 2020;18(08): 2064-2065

49 Tang N. Response to "lupus anticoagulant is frequent in patients with Covid-19" (JTH-2020-00483). J Thromb Haemost 2020;18 (08):2065-2066

50 Gatto M, Perricone C, Tonello M, et al. Frequency and clinical correlates of antiphospholipid antibodies arising in patients with SARS-CoV-2 infection: findings from a multicentre study on 122 cases. Clin Exp Rheumatol 2020;38(04):754-759

51 Siguret V, Voicu S, Neuwirth M, et al. Are antiphospholipid antibodies associated with thrombotic complications in critically ill COVID-19 patients? Thromb Res 2020;195:74-76

52 Fan S, Xiao M, Han F, et al. Neurological manifestations in critically ill patients with COVID-19: a retrospective study. Front Neurol 2020;11:806

53 Devreese KMJ, Linskens EA, Benoit D, Peperstraete H. Antiphospholipid antibodies in patients with COVID-19: a relevant observation? J Thromb Haemost 2020;18(09):2191-2201

54 Pineton de Chambrun M, Frere C, Miyara M, et al. High frequency of antiphospholipid antibodies in critically ill COVID-19 patients: a link with hypercoagulability? J Intern Med 2021;289(03): 422-424

55 Reyes Gil M, Barouqa M, Szymanski J, Gonzalez-Lugo JD, Rahman $\mathrm{S}$, Billett $\mathrm{HH}$. Assessment of lupus anticoagulant positivity in patients with coronavirus disease 2019 (COVID-19). JAMA Netw Open 2020;3(08):e2017539

56 de Ocáriz XGL, Castro Quismondo N, Vera Guerrero E, Rodríguez Rodríguez M, Ayala Díaz R, Martínez López J. Thrombosis and antiphospholipid antibodies in patients with SARS-COV-2 infection (COVID-19). Int J Lab Hematol 2020;42(06):e280-e282

57 Cuenca Saez MA, Gomez-Biezna SL. Immunoglobulin A antiphospholipid antibodies in patients with Chilblain-like lesions during the COVID-19 pandemic. Actas Dermosifiliogr 2021;112(03): 290-292

58 Tvito A, Ben-Chetrit E, Zimmerman FS, Asher E, Helviz Y. Lupus anticoagulant in patients with COVID-19. Int J Lab Hematol 2021; 43(01):e17-e18

59 Ferrari E, Sartre B, Squara F, et al. High prevalence of acquired thrombophilia without prognosis value in patients with coronavirus disease 2019. J Am Heart Assoc 2020;9(21):e017773

60 Zhang Y, Cao W, Jiang W, et al. Profile of natural anticoagulant, coagulant factor and anti-phospholipid antibody in critically ill COVID-19 patients. J Thromb Thrombolysis 2020;50(03):580-586

61 Tan YK, Goh C, Leow AST, et al. COVID-19 and ischemic stroke: a systematic review and meta-summary of the literature. J Thromb Thrombolysis 2020;50(03):587-595

62 Xiao M, Zhang Y, Zhang S, et al. Antiphospholipid antibodies in critically ill patients with COVID-19. Arthritis Rheumatol 2020;72 (12):1998-2004

63 Fan BE, Ng J, Chan SSW, et al. COVID-19 associated coagulopathy in critically ill patients: a hypercoagulable state demonstrated by parameters of haemostasis and clot waveform analysis. J Thromb Thrombolysis 2021;51:663-674

64 Gazzaruso C, Mariani G, Ravetto C, et al. Lupus anticoagulant and mortality in patients hospitalized for COVID-19. J Thromb Thrombolysis 2020 (ePub ahead of print). Doi: 10.1007/s11239-02002335-w

65 Le Joncour A, Frere C, Martin-Toutain I, et al. Antiphospholipid antibodies and thrombotic events in COVID-19 patients hospitalized in medicine ward. Autoimmun Rev 2021;20(02):102729

66 Bauer W, Galtung N, Neuwinger N, et al. A matter of caution: coagulation parameters in COVID-19 do not differ from patients with ruled-out SARS-CoV-2 infection in the emergency department. TH Open 2021;5(01):e43-e55 
67 Hamadé A, Woehl B, Harzallah I, Talbot M, Tousch J, Jambert L. Antiphospholipid antibodies in patients with coronavirus disease 2019 infection hospitalized in conventional unit. Blood Coagul Fibrinolysis 2021;32(02):73-79

68 Karahan S, Erol K, Yuksel RC, Artan C, Celik I. Antiphospholipid antibodies in COVID-19-associated pneumonia patients in intensive care unit. Mod Rheumatol 2021 (ePub ahead of print). Doi: 10.1080/14397595.2021.1892257

69 Beyrouti R, Adams ME, Benjamin L, et al. Characteristics of ischaemic stroke associated with COVID-19. J Neurol Neurosurg Psychiatry 2020;91(08):889-891

70 Galeano-Valle F, Oblitas CM, Ferreiro-Mazón MM, et al. Antiphospholipid antibodies are not elevated in patients with severe COVID-19 pneumonia and venous thromboembolism. Thromb Res 2020;192:113-115

71 Devreese KM. Antiphospholipid antibody testing and standardization. Int J Lab Hematol 2014;36(03):352-363

72 Schouwers SM, Delanghe JR, Devreese KM. Lupus anticoagulant (LAC) testing in patients with inflammatory status: Does Creactive protein interfere with LAC test results? Thromb Res 2010;125(01):102-104

73 De Kesel PMM, Devreese KMJ. The effect of unfractionated heparin, enoxaparin, and danaparoid on lupus anticoagulant testing: Can activated carbon eliminate false-positive results? Res Pract Thromb Haemost 2019;4(01):161-168

74 Tripodi A, Cohen H, Devreese KMJ. Lupus anticoagulant detection in anticoagulated patients. Guidance from the Scientific and Standardization Committee for lupus anticoagulant/antiphospholipid antibodies of the International Society on Thrombosis and Haemostasis. J Thromb Haemost 2020;18(07):1569-1575
75 Favaloro EJ, Bonar R, Marsden K. Internal quality control and external quality assurance in testing for antiphospholipid antibodies: Part II-Lupus anticoagulant. Semin Thromb Hemost 2012;38(04):404-411

76 Abdel-Wahab N, Talathi S, Lopez-Olivo MA, Suarez-Almazor ME. Risk of developing antiphospholipid antibodies following viral infection: a systematic review and meta-analysis. Lupus 2018;27 (04):572-583

77 Abdel-Wahab N, Lopez-Olivo MA, Pinto-Patarroyo GP, SuarezAlmazor ME. Systematic review of case reports of antiphospholipid syndrome following infection. Lupus 2016;25(14): 1520-1531

78 Cavalli E, Bramanti A, Ciurleo R, et al. Entangling COVID-19 associated thrombosis into a secondary antiphospholipid antibody syndrome: diagnostic and therapeutic perspectives (Review). Int J Mol Med 2020;46(03):903-912

79 Million M, Bardin N, Bessis S, et al. Thrombosis and antiphospholipid antibody syndrome during acute $Q$ fever: a cross-sectional study. Medicine (Baltimore) 2017;96(29):e7578

80 Mendoza-Pinto C, García-Carrasco M, Cervera R. Role of infectious diseases in the antiphospholipid syndrome (including its catastrophic variant). Curr Rheumatol Rep 2018;20(10):62

81 Catoggio C, Alvarez-Uría A, Fernandez PL, Cervera R, Espinosa G. Catastrophic antiphospholipid syndrome triggered by fulminant disseminated herpes simplex infection in a patient with systemic lupus erythematosus. Lupus 2012;21(12):1359-1361

82 Favaloro EJ, Gilmore G, Arunachalam S, Mohammed S, Baker R. Neutralising rivaroxaban induced interference in laboratory testing for lupus anticoagulant (LA): a comparative study using DOAC Stop and andexanet alfa. Thromb Res 2019;180:10-19 